\title{
OAW
}

Österreichische Akademie

der Wissenschaften

\section{Call TA'11}

\section{Dezember 2010}

Converging

Technologies

Social Network

Sites

Interdisziplinarität und Technowissenschaften 



\section{Editorial}

\section{Werte LeserInnen!}

Neben den jährlichen Tagungen des ITA im Frühsommer in Wien - auf den druckfrischen Call for Papers zur TA'1 1 am 20. Juni 2011 sei hiermit hingewiesen (siehe Seite 2) - stellen die alle zwei Jahre statffindenden Konferenzen der NTA einen Fixpunkt im Kalender der deutschsprachigen TA-Gemeinde dar. Die vierte Tagung des Netzwerks Technikfolgenabschätzung (NTA4) fand vom 24.-26. November in Berlin statt. Eingangs kommentierte und interpretierte ein hochkarätig besetztes Podium die Erfolgsgeschichte des Büros für TA beim Deutschen Bundestag, das im Herbst 20 Jahre alt geworden war. 150 TeilnehmerInnen, 36 Vorträge in drei Parallelsessions und eine Postersession mit 24 Beiträgen zeigten sowohl die Breite, als auch den Reifegrad, den die TA im deutschsprachigen Raum bereits erreicht hat. Unter den Keynote-Vortragenden waren die Gallionsfigur der niederländischen Wissenschafts- und Technikforschung, Arie Rip, und der Staatssekretär a.D. im deutschen Forschungsministerium, Frieder MeyerKrahmer. Durch die Breite der Beiträge wurde Rips rhetorische Frage "TA never dies?" nicht nur von ihm selbst - freilich mit einigen Aufträgen an die Community garniert - positiv beantwortet. Der programmatische Anspruch, einen „Systemblick auf die Technikentwicklung" zu werfen, wurde eindrucksvoll eingelöst: Die TA selbst, aber auch verwandte Ansätze wie Ökobilanzierung und Nachhaltigkeitsforschung schauen über den berühmten disziplinären Tellerrand und müssen dabei zahllose organisatorische, methodische und kommunikative Herausforderungen meistern. Dass das so entstehende Wissen auch dort ankommt, wo es wirksam werden soll (in der Politik), ist freilich höchst voraussetzungsvoll, wie Meyer-Krahmer anhand von Praxisbeispielen nachwies - nicht ohne die Rolle der TA als treibende Kraft zu unterstreichen.

Mit Freude darf ich hier noch berichten, dass sich unser geschätzter Kollege Alexander Bogner diesen Oktober im Fach Soziologie habilitiert hat.

Ich wünsche Ihnen frohe und geruhsame Tage rund um den Jahreswechsel bei der Lektüre der letzten Neuigkeiten aus dem ITA und der TA. Szene.

Michael Nentwich

\section{Inhalt}

\section{ITA-Projekte}

Partizipation in Technikfragen das Thema der $\operatorname{TA}^{\prime} 11$ 2

NanoTrust:

Neue Herausforderungen bis 2014 3

"Making Perfect Life?" -

Präsentation im Europäischen Parlament..........4

Österreich in der neuen Eurobarometer-

Umfrage zur Biotechnologie 5

Beteiligung von BürgerInnen an lokaler Klimaschutzpolitik 6

Pilotstudie Converging Technologies ................7

Social Network Sites -

ein Trend auch in der Wissenschaft? .9

\section{TA-aktuell}

Dekonstruktion und (Ver-)Lernen in der Softwareentwicklung.

Technologiespezifische Perspektiven auf digitale Pornografie

Interdisziplinarität und Technowissenschaft ......12

Technology Governance:

Wie kann TA dazu effektiv beitragen?

Geschichte der Nanotechnologie -

Bericht von der 2. S.NET-Konferenz

Technikfolgenabschätzung international:

EPTA-Jahrestagung 2010

Risikobewertung und Risikomanagement der Nanotechnologien

Lernen für die Transformation: Neue

Perspektiven in der technischen Ausbildung..... 19

Aktuelle Publikationen .20

ITA-Veranstaltungen 25

Kontakt

Impressum 


\section{Partizipation in Technikfragen - das Thema der TA'11}

\begin{abstract}
Stellt Partizipation in Technikfragen heute eine legitime Hoffnung dar - oder ist sie bloße Illusion? Dieser brisanten Fragestellung wird sich die elfte internationale TA-Konferenz des ITA am 20. Juni 2011 in Wien widmen.
\end{abstract}

Es scheint paradox: In einer Zeit nachlassender Partizipationsansprüche in Technikfragen versucht die Politik verstärkt, Interesse zu wecken und Partizipation zu stimulieren. Wissenschaftsschiffe werden auf Reisen geschickt, um die Bürgerlnnen für die Energiewende ins Boot zu holen; Nano-Trucks fahren durchs Land, um frühzeitig Debatten über eine Hoffnungstechnologie zu initiieren; Bürgerkonferenzen werden organisiert, um der Bevölkerung in Sachen Klimawandel oder Stammzellforschung eine Stimme zu geben.

Die TA hat eine Reihe von Verfahren entwickelt, um gesellschaftliche Gruppen zu beteiligen, die ansonsten nicht in Prozesse der Technikbewertung involviert sind. Damit verbindet sich die Erwartung, alternative Rationalitäten, Interessen und Werte sichtbar zu machen, sozial robuste Lösungen zu finden, die Legitimität von Entscheidungen zu erhöhen und Teilhabemöglichkeiten zu erweitern, um damit die Demokratiequalität zu verbessern.

Die partizipativen Ansätze in der Technikfolgenabschätzung (pTA) sind nicht ohne Kritik geblieben, die aber bisher kaum systematisch und auf Basis haltbarer empirischer Analysen entwickelt wurde. Deshalb existieren nur wenige belastbare Aussagen über Nutzen und Grenzen einzelner Partizipationsformate und kaum Antworten auf die Frage nach einer Alternative zu Partizipation.

Die TA'1 1 zielt darauf ab, den Stellenwert von Partizipation in Technikfragen neu zu bestimmen und ein stimmiges Bild über Ausmaß, Formen, Nutzen und Grenzen von pTA zu entwickeln. Nur so sind begründete Warnungen oder positive Empfehlungen für pTA im Einzelfall möglich. Die $T^{\prime} 11$ wird sich daher besonders folgenden Fragen widmen:
Welche tragfähigen Legitimationsbegründungen gibt es für Partizipation in Technikfragen? Welcher Anspruch auf Legitimität lässt sich mit welchen Verfahren der pTA verbinden? Wo ergeben sich Grenzen der Legitimation?

Was leisten Verfahren, die primär auf Interessenabstimmung und Erzeugung von neuem Wissen abzielen, etwa in der partizipativen Technikgestaltung oder der konstruktiven TA? In welchem Verhältnis steht die Laienexpertise zu anderen Wissensformen und wie werden diese integriert?

Mit welchen unterschiedlichen, oft impliziten Zielvorstellungen wird pTA durchgeführt? Welche Erwartungen haben die beteiligten AkteurInnen? Welchen Nutzen hat pTA für Politik, Wirtschaft, Forschung, den öffentlichen Diskurs, die TA selbst?

Welchen Status und welche Rolle hat pTA in verschiedenen Ländern? Welchen Einfluss haben unterschiedliche "politische Kulturen" für die Praxis der pTA? Lassen sich die in einem bestimmten politischen Kontext entwickelten Verfahren erfolgreich übertragen?

Welche Bedeutung haben elektronische Medien für die politische Beteiligung von Bürgerlnnen (e-participation)? Welche Potenziale haben diese neuen Beteiligungsformen für die TA?

Welche Konsequenzen hat es für das Selbstverständnis der TA, wenn sie verstärkt als Organisator von Partizipationsevents in Erscheinung tritt?

Diese und ähnliche Fragen wollen wir im Rahmen unser nächstiährigen TA-Konferenz diskutieren und vertiefen.

Einreichung von Abstracts im Ausmaß von ca. 500 Wörtern bitte bis 4 . Februar ' 11 per E-Mail antamail@oeaw.ac.at.

Konferenzhomepage: www.oeaw.ac.at/ita/ta11.

Alexander Bogner, Michael Ornetzeder, Helge Torgersen 


\section{NanoTrust: \\ Neue Herausforderungen bis 2014}

\begin{abstract}
Angesichts der anhaltenden Entwicklung im Bereich Nanotechnolgien hat sich das BMVIT nach einer positiven Stellungnahme des NanoTrust-Beirats, zweier externer wissenschaftlicher Gutachter und intensiver interner Beratung entschlossen, die Laufzeit des Projekts NanoTrust bis April 2014 zu verlängern. Das ITA erhält damit die Möglichkeit, dieses spannende Projekt fortzusetzen und weiterzuentwickeln.
\end{abstract}

Hauptaufgabe des Projekts wird weiterhin die kontinuierliche Herausforderung sein, den Stand des Wissens zu möglichen Gesundheits- und Umweltwirkungen zu erheben, zu analysieren und für die Politik, die betroffene Verwaltung, die involvierte Forschungscommunity und die interessierte Öffentlichkeit aufzubereiten. Dazu kommt in den kommenden Jahren die intensivierte Beschäftigung mit Fragen des ArbeitnehmerInnen- und VerbraucherInnenschutzes, mit der Regulierung dieses Bereichs sowie mit dem Diskurs um die Nanotechnologie.

Neben Beiträgen zur Fachliteratur publiziert NanoTrust die auch international als richtungweisend anerkannten NanoTrust-Dossiers. Die Dossiers stellen aktuelle Themen in zugänglicher Sprache auf maximal sechs Seiten dar. Bereits das 21. in dieser Reihe wird demnächst erscheinen. Aufgrund der großen Nachfrage werden die Dossiers ab sofort auch auf Englisch erscheinen, um sie in die internationalen Netzwerke und Informationssysteme einspeisen zu können. Seit November erscheinen sukzessive auch englische Fassungen der bereits seit 2008 herausgegebenen Dossiers, teilweise wo notwendig auch mit kleinen Aktualisierungen.

Die kommende Projektphase wird nicht zuletzt durch die aktive Mitwirkung an der Implementierung des österreichischen Nano-Aktionsplans (NAP) geprägt sein, zu dem NanoTrust aufgrund seiner spezifischen Stellung und Ausstattung beitragen kann. Zum einen werden wir eine beratende Funktion im Zusammenhang mit den vorgesehenen Fördermitteln für die naturwissenschaftliche Begleitforschung (Umwelt-, Gesundheits- und Sicherheitsfragen - EHS) ausüben. Dieser sog. EHS-Fonds unter Federfüh- rung des Umweltministeriums, wesentlich dotiert durch das BMVIT, wird in den nächsten Jahren Forschung unterstützen, die Wissenslücken etwa im Bereich der Toxikologie füllen oder Nachweismethoden entwickeln soll.

Zum anderen wirkt das NanoTrust-Team mit seinem Überblickswissen am Aufbau der unter Federführung des Gesundheitsministeriums entstehenden Nano-Informationsplattform (NIP) mit. Die NIP dient dem kontinuierlichen Austausch zu aktuellen Fragen des Umgangs mit Nanotechnologien zwischen den befassten Behörden und der Wissenschaft. Darüber hinaus wird in diesem Rahmen eine Schnittstelle zur Information der und Kommunikation mit der interessierten Öffentlichkeit geschaffen.

Die NanoTrust-Projektmitarbeiterlnnen können in Phase 2 bereits auf ein engmaschiges Netzwerk an Kooperationspartnerlnnen und ExpertInnen im In- und Ausland zurückgreifen, wenn es darum geht, das vorhandene Wissen zu Chancen und Risiken sowie zum bestmöglichen Umgang mit den Nanotechnologien für Österreich verfügbar zu machen. Insbesondere die internationalen Netzwerke der EU-geförderten Aktivitäten DaNa, ObservatoryNANO, NanoImpactNet und ERA-NET SIINN werden neben den direkten Kontakten zum ITAS in Karlsruhe, zu SCENIHR in Brüssel, zur OECD in Paris, zum JRC in Ispra oder zur EMPA in Zürich eine wichtige Rolle spielen. Diese fruchtbaren Beziehungen haben bereits zu Kooperationen geführt, etwa bei der laufenden Studie zu "NanoSafety" für das Europäische Parlament (STOAPanel) und bei einem Dreiländervergleich Deutschland-Schweiz-Österreich der "Nanopolitiken" (siehe dazu auch Seite 4). NanoTrust wird mit großer Wahrscheinlichkeit auch in den kommenden Jahren immer wieder als Kooperationspartner in derartigen international akkordierten Aktivitäten angefragt werden.

Die Fortsetzung der Reihe der NanoTrust-Herbsttagungen (siehe dazu auch Seite 17), bei der internationale ExpertInnen ihr Wissen der heimischen Fachwelt zur Verfügung stellen, ist ebenso vorgesehen, wie eigene Beiträge zu internationalen Konferenzen und Treffen (z. B. auf der S.NET, siehe Seite 14). 
Darüber hinaus wird das NanoTrust-Team ab dem Wintersemester 2011/12 im neven interuniversitären Masterlehrgang zu "Nanobiowissenschaften und Nanomedizin" das Modul 4 zu Risiken und regulatorischen Aspekten der $\mathrm{Na}$ notechnologie anbieten.
Weiter Informationen finden sich im Internet unter nanotrust.ac.at ebenso wie Erklärungen und nähere Informationen zu den oben genannten Institutionen und Projekten (siehe NanoTrust-Linksammlung).

Michael Nentwich

\section{"Making Perfect Life?" Präsentation im Europäischen Parlament}

\begin{abstract}
Welche Entwicklungen im Bereich „Bioengineering" werden uns in den nächsten Jahrzehnten beschäftigen? Das ETAGProjekt „Making Perfect Life?" versucht, darauf eine Antwort zu geben, die sich nicht nur in technischen Vorhersagen erschöpft, sondern Entwicklungstendenzen aufzuzeigen versucht. Vom niederländischen Rathenau-Instituut koordiniert, beschäftigt sich das Konsortium mit den Auswirkungen des Zusammenwachsens von Biologie und Technologie in den Bereichen regenerative Medizin, synthetische Biologie (vom ITA betreut), Hirnforschung und künstliche Intelligenz.
\end{abstract}

Organisiert vom TA-Büro des Europäischen Parlaments (STOA), wurde der Bericht zum ersten Projektteil am 11.11.2010 im Europäischen Parlament unter Leitung der Abgeordneten Malcom Harbour und Vittorio Prodi diskutiert. Jeweils vier internationale Diskutantlnnen behandelten in drei Round-Tables die Aspekte "How biology is becoming technology", "How technology is becoming biology" und "Political and policy implications of bio-engineering in the $21^{\text {st }}$ century".

Dem zahlreich erschienenen Publikum wurde so ein Kaleidoskop von zukünftigen Entwicklungen und den damit verbundenen Herausforderungen auf dem Gebiet der "Converging Technologies" präsentiert. Es fragte sich allerdings, inwieweit ein notwendigerweise spekulativer Vorgriff auf die Zukunft Parlamentsabgeordneten noch als unmittelbar policy-relevant erschien.

Unter dem Motto "Biology becoming technology" sah Antoine Danchin (Univ. Hong Kong) in der synthetischen Biologie eine stark reduktionistische Tendenz. Gene seien aber keine simplen Blaupausen, sondern Instrumente zur Aufnahme von Information aus der Umwelt, das Verhalten von Organismen schwer vorhersagbar. Gefährlicher als Neukonstruktion sei daher die bloße Nachahmung der Natur - ein Thema, das Andrew Webster (Univ. York) mit Hinweis auf die Do-lt-Yourself-biology-Bewegung (DIYbio) aufgriff. Veerle Visser-Vandewelle (Maastricht UMC) meinte dagegen, dass es kaum DIY-Neurobiologie geben wird; Probleme rührten eher aus ungleicher Verteilung. Stephen Minger (GE Healthcare) schließlich sah - etwa bei der Prüfung von Stammzell-Therapien - Bedarf nach Unsicherheits-toleranten Policy-Optionen.

Unter "Technology becoming biology" wies Brigitte Krenn (OFAI Wien) auf die Möglichkeit immer realistischerer Simulationen hin, unterstütz† von Anders Lansner (Univ. Stockholm). Auch Mark Bedau (Reed College, Portland) sieht rasanten Fortschritt, für ihn leben Artefakte der synthetischen Biologie „buchstäblich." Jutta Weber (TU Braunschweig) stand hingegen der behaupteten Verbindung von Technologie und Biologie skeptisch gegenüber - erstere sei lediglich „inspiriert", Nutzerlnnen etwa könnten Unterschiede durchaus erkennen. Sie warnte auch vor einer unkritischen Verwendung des Begriffs "Emergenz", während Mark Bedau das Entstehen emergenter Eigenschaften als Teil dessen ansah, was Technologie leisten können wird. Der regulatorische Umgang mit derartigen Artefakten bleibt freilich eine Herausforderung.

Bezüglich Auswirkungen auf die Politik blieb die Debatte allerdings eher im Abstrakt-Philosophischen. Nach einer Diskussion über Herausforderungen für das Prinzip der Würde durch neue 
Entwicklungen (Judit Sandor, Central European University, Budapest und Inez de Beaufort, Erasmus Universität Rotterdam) kritisierte Roger Strand (Univ. Bergen) den Begriff "Megatrend", mit dem der Bericht die Technologie/BiologieVerbindung bezeichnete - als ob diese unabhängig vom politischen Willen wäre. Andrea Bonaccorsi warnte vor einem Hype und forderte die Verantwortung von Wissenschaft und Technologie ein. Die "Upstream"--Orientierung von ELSI-Studien ${ }^{1}$ und TA, für die sich Strand aussprach, zeigte aber an diesem Tag ihre Grenzen: Nur wenige Abgeordnete waren anwesend, zu fern schien das Thema dem täglichen Politikgeschehen.
Letztlich diente die Veranstaltung auch der Demonstration, wie weit Technikentwicklung, Politik und wissenschaftliche Beschäftigung auseinander driften können. Obwohl an diesem Nachmittag Themen diskutiert wurden, die in naher Zukunft hoch relevant werden können, schien doch die parallel statffindende Abstimmung zum Europäischen Binnenmarkt wesentlich politikrelevanter.

\footnotetext{
1 "Ethical, Legal and Social Issues (ELSI)" Studien/Human Genome Project.
}

Helge Torgersen

\section{Österreich in der neuen Eurobarometer-Umfrage zur Biotechnologie}

\begin{abstract}
Etwa alle fünf Jahre lässt die Europäische Kommission die öffentliche Wahrnehmung der Biotechnologie repräsentativ erheben; das ITA ist seit fast zwei Jahrzehnten daran beteiligt. Die jüngste Umfrage wurde erstmals in 32 Ländern Europas einschließlich der Türkei durchgeführt.
\end{abstract}

Gegenüber 2005 zeigen die Resultate, dass bezüglich einer Reihe von modernen Technologien das Misstrauen in Regierungen und Industrie abgenommen hat. Nutzen, Sicherheit und Nachhaltigkeit werden heute differenzierter betrachtet, technisch weniger tief greifende Alternativen (z. B.: Gentechnik mit arteigenen Genen) eher akzeptiert. Gefordert wird eine Regulierung im öffentlichen Interesse sowie Mitsprache der Bevölkerung, wenn es um Werte geht, die als gesellschaftlich wichtig gelten. Die Prioritäten unterscheiden sich allerdings innerhalb Europas.

Auf die Frage, ob eine Technologie in den nächsten 20 Jahren "das Leben verbessern" wird, verlieren Bio- und Nanotechnologie etwas, auch Computer/Kommunikationstechnologien stagnieren. Technologien zur Energieerzeugung gewinnen, besonders Solar- und Windenergie; Kernenergie wird weniger negativ beurteilt.
Im europäischen Vergleich nimmt Österreich eine Sonderstellung ein. Obwohl auch hier die positiven Erwartungen stark überwiegen, scheint man in keinem anderen Land Europas auf so breiter Front skeptisch zu sein. In Österreich sind nicht nur die Erwartungen an Biotechnologie oder Kernenergie besonders niedrig, sondern auch an die Raumfahrt oder an Computer. Österreich teilt mit Griechenland den vorletzten Platz bezüglich Nanotechnologie. Nur bei Wind- und Solarenergie liegt Österreich im oberen europäischen Mittelfeld.

Bei Biomedizin zeigen sich die Österreicherlnnen besonders reserviert: Weder Hirnforschung noch synthetische Biologie finden annähernd Zustimmungwerte wie im europäischen Schnitt. Stammzellforschung wird zwar mancherorts kritischer gesehen als 2005, in Österreich aber am stärksten abgelehnt.

Die Grüne Gentechnik ist erwartungsgemäß wenig beliebt. Die niedrigen Zustimmungswerte der Österreicherlnnen für gentechnisch veränderte Nahrungsmittel bewegen sich im (unteren) europäischen Mittelfeld; es scheint, als hätte die Öffentlichkeit in anderen Ländern inzwischen eine ähnlich ablehnende Haltung. Bio-Treibstoffe werden nur in vier Ländern skeptischer beurteilt. 
Auch was das Klonen von Nutztieren angeht, ist die Akzeptanz in vielen Ländern ähnlich niedrig. Ähnlich wie in Deutschland und der Schweiz sollen eher moralische als wissenschaftliche Beurteilungskriterien gelten. Obwohl das Vertrauen in Wissenschaft, Regulierung und Industrie gestiegen ist, soll die Öffentlichkeit und nicht Expertlnnen über den Einsatz entscheiden.

Auffällig ist, dass die Zustimmung in Österreich in vielen Fragen niedrig ist. Geringere Zustimmung findet sich meist nur in südosteuropäischen Ländern. Oft sind aber die Unterschiede weniger groß als eine Reihung suggeriert. Die genauere Analyse zeigt etwa, dass in Österreich mehr Befragte negative Effekte von Biotechnologie oder Kernenergie erwarten als in anderen Ländern. Computern oder Raumfahrt hingegen werden weder positive noch negative Auswirkungen zugeschrieben.

Auf der Suche nach möglichen Gründen fällt auf, dass sich Österreicherlnnen schlechter über die jeweiligen Technologien informiert fühlen als andere, das gilt auch für Gentechnik in Nahrungsmitteln. Ähnliche Muster finden sich in südosteuropäischen Ländern und in Portugal.

Ein Zusammenhang von Technikablehnung aus Furcht und geringem Informationsstand ist aber nicht ersichtlich. Wissenschaft und Technik werden in Österreich nicht als bedrohlich wahrgenommen und auch mit Hoffnungen (Gesundheit, Wohlstand, berufliches Vorankommen) verbunden. Allerdings scheinen sie nicht als Teil der Kultur zu gelten und einen geringen Stellenwert zu haben. Der Eindruck besonderer Technologiekritik in Österreich mag also täuschen - offenbar ist vielen das Thema einfach egal.

\section{Eurobarometer:}

ec.europa.eu/public_opinion/archives/

eb special en.htm\#341, Konsortialbericht von STEPE:

ec.europa.eu/public opinion/archives/ebs/ ebs_341_winds_en.pdf.

Helge Torgersen

\section{Beteiligung von Bürgerlnnen an lokaler Klimaschutzpolitik}

\begin{abstract}
Das ITA untersucht im europäischen Forschungsprojekt „Environmental Electronic Democracy" (e2democracy) die Effekte unterschiedlicher Beteiligungsangebote - online und offline - auf Engagement und Verhalten im Hinblick auf den Klimaschutz, insbesondere auf Energieeinsparung und $\mathrm{CO}_{2}$-Reduktion.
\end{abstract}

Im Zentrum des Projekts stehen folgende Fragen:

- Welche Rolle spielt E-Partizipation bei Klimaschutzstrategien auf lokaler Ebene?

- Welche Effekte auf umweltbezogene Einstellungen und Verhaltensweisen von Bürgerlnnen sowie auf die lokale Nachhaltigkeitspolitik zeichnen sich ab?

- Welche Kontextfaktoren sind für Nutzung und Auswirkungen von E-Partizipation im nationalen sowie im länderübergreifenden Vergleich relevant?
Die Ergebnisse von repräsentativen Umfragen, die zu Projektbeginn durchgeführt wurden, deuten auf eine generell hohe Bereitschaft zur aktiven Beteiligung im Klimaschutz hin. In Österreich sehen sich in beiden Untersuchungsregionen (Bregenz und Mariazeller Land) über $90 \%$ der Befragten selbst in der Pflicht, einen großen bis sehr großen Beitrag zur $\mathrm{CO}_{2}$ - $\mathrm{Re}$ duktion zu leisten. Knapp 70 \% sind zum Monitoring ihrer Einsparbemühungen durch die individuelle $\mathrm{CO}_{2}$-Bilanzierung bereit. Zugleich hält eine breite Mehrheit die gemeinsamen Anstrengungen aller Akteurlnnen für zielführend. Die Motivation nimmt zu, wenn auch öffentliche Einrichtungen und Unternehmen an der $\mathrm{CO}_{2}$-Bilanzierung teilnehmen.

Im Oktober 2010 hatten die Klimadialoge von e2democracy in den Partnerländern Deutschland, Österreich und Spanien unterschiedliche Laufzeiten hinter sich. Insgesamt zeigt sich mittlerweile ein Anwachsen des Engagements auf einen Gesamtstand von 730 registrierten Teil- 
nehmerlnnen, von denen 420 (knapp $60 \%$ ) kontinuierlich aktive Beiträge leisten. Die aktiv involvierten Bürgerlnnen aus Spanien stellen mit derzeit 280 die bedeutendste Gruppe und verzeichnen die stärksten Zuwächse. In Österreich entfällt von den über 120 Anmeldungen der etwas größere Anteil auf Bregenz, der kleinere auf das Mariazellerland. Die Kerngruppe der kontinuierlich aktiv Beteiligten erreicht in Österreich zwar nicht ganz den Spitzenwert Spaniens (fast $70 \%$ ), immerhin aber einen gröBeren Anteil als in Bremen (unter 40 \%). Bisher fällt auf, dass die Gruppe der mit traditionellen Medien Teilnehmenden (die "Offliner") in hohem Ausmaß von Beginn an stabil und aktiv dabei ist, während die Gruppe der über Internet Beteiligten (die "Onliner") nicht das gleiche Ausmaß an Kontinuität und aktiven Beiträgen aufweist.

Bisher konzentrierten sich die Partizipationsprozesse in allen Regionen weitgehend auf Maßnahmen der CO2-Bilanzierung. In Workshops und durch Informationsmaterialien konnten die TeilnehmerInnen die Komponenten OnlineRechner und $\mathrm{CO}_{2}$-Haushaltsbuch kennenlernen. Alle Beteiligten erhalten einen regelmäBigen Newsletter sowie verschiedene Tipps zum Energiesparen und Klimaschutz, die über die
Internetseite des Projekts oder das $\mathrm{CO}_{2}$-Haushaltsbuch angeboten werden. Nach jeder abgeschlossenen Erhebung (alle zwei Monate) stehen Auswertungen zur Verfügung, die Rückschlüsse über die jeweils eigene $\mathrm{CO}_{2}$-Bilanz sowie den Vergleich mit Durchschnittswerten anderer Teilnehmerlnnen ermöglichen. In der nächsten Phase können die Bürgerlnnen ihre Erfahrungen untereinander austauschen und erhalten weitere gezielte Informations- und Beratungsangebote. Verstärktes Augenmerk soll auch den Klimaschutzmaßnahmen von Unternehmen und einzelnen Verwaltungsstellen gewidmet werden.

Inwieweit die im Rahmen des Projektes gesetzten Maßnahmen, insbesondere die $\mathrm{CO}_{2}$-Bilanzierung, zu nachhaltig wirksamen Effekten führen können, ist zu diesem Zeitpunkt nicht absehbar. Allerdings sind bestimmte soziale Lerneffekte oder zumindest ein gesteigertes Bewusstsein für Umweltbelange bei den beteiligten Akteurlnnen zu erwarten. Dies ist eine wesentliche Voraussetzung dafür, das politische System zu verstärkten Maßnahmen für den Klimaschutz zu bewegen.

Projekthomepage: e2democracy.eu.

Georg Aichholzer, Stefan Strauß

\section{Pilotstudie Converging Technologies}

\begin{abstract}
Im Auftrag des BMVIT wurden im ITAProjekt "Converging Technologies (CT)" allgemeine Rahmenbedingungen für Forschungs- und Entwicklungsmöglichkeiten der CT in Österreich untersucht. Von den sogenannten konvergierenden Technologien spricht man im Zusammenhang mit der Hypothese, dass die Bio-, Nano-, Info- und Kognitionstechnologien in gewisser Weise zusammenwachsen könnten.
\end{abstract}

Die Ausführung der Studie lag bei der Forschungsgruppe IDC, das ITA war für Koordination und Projektleitung zuständig, die Ergebnisse werden im Jänner 2011 präsentiert. Ziele der Studie waren (1) die Sondierung und Abbildung des Begriffsfeldes der Converging Technologies sowie eine Analyse unterschiedlicher Begriffsdefinitionen zu CT, (2) die Erhe- bung europäischer Aktivitäten zur F\&E in diesem Bereich und (3) die Identifikation möglicher Interessenten, Ansprechpartnerlnnen und Themenfelder in Österreich im Hinblick auf eine mögliche Etablierung einer wissenschaftlichen CT-Community.

Zunächst wurde der Versuch unternommen, den Bedeutungsumfang und die Funktionsbreite des Begriffs "Converging Technologies" und verwandter Ausdrücke (NBIC - Nano/Bio/Info/ Cogno, GRN, GRIN, GRAIN, etc.) abzuschätzen und einzugrenzen. In den folgenden Arbeitspaketen stand die Frage im Vordergrund, welche Art von Projekten unter diesem Stichwort tatsächlich für förderungswürdig befunden wurden und welche Disziplinen hier beteiligt waren. Diese Fragen wurden auf europäischer Ebene erhoben und analysiert. Um eine Übersicht über bereits abgeschlossene oder laufen- 
de Forschungsvorhaben zur CT im internationalen Kontext zu erlangen, wurden bisherige Ausschreibungen und Projekte im FP6 und FP7, ERA-NET etc. analysiert, wobei sich die Kernfragen auf die beteiligten Fachdisziplinen, ihre RepräsentantInnen und die jeweiligen Forschungsherausforderungen bezogen. In einem abschlieBenden Workshop wurden mit ausgewählten Forscherlnnen der "CT-Szene" die Ergebnisse aus den ersten Erhebungen diskutiert, um ein besseres Verständnis für Probleme und Herausforderungen in diesem Bereich zu gewinnen.

\section{Europäische Forschungsaktivitäten}

Der Begriff "Converging Technology" taucht seit 2001 häufig in wissenschaftlichen Publikationen auf; bedeutend mehr wird freilich zu den einzelnen NBIC-Teilbereichen publiziert, vor allem zu Nanotechnologie und Biotechnologie sowie zu "Nano-Biotechnologie". Im 6. und 7. EUForschungsrahmenprogramm wurden F\&E-Projekte mit CT-Bezug in erster Linie innerhalb des Programms "Nanowissenschaften und -technologien, Materialien und Produktion (NMP)" ausgeschrieben. Der Fokus wurde hier auf den Bereich Nanotechnologie gelegt und die Begriffe "Converging Technologies" sowie "Converging Sciences" und "Convergence of Technologies" relativ weit gefasst. So wird auch die Konvergenz anderer Wissenschafts- und Technologiebereiche, wie der Physik, der Chemie oder der Mathematik berücksichtigt.

Verglichen mit den F\&E-Projekten wurde von der EU eine weitaus größere Anzahl von sozialwissenschaftlichen Projekten gefördert, die sich mit gesellschaftlichen Auswirkungen von CT beschäftigen. Dies mag damit zusammenhängen, dass einige Anwendungsbereiche von CT direkte Auswirkungen für den Menschen haben können, etwa im medizinischen Bereich, aber auch die Befürchtung widerspiegeln, es könnte eine ähnliche öffentliche Ablehnung wie bei der grünen Gentechnik geben. Viele der Begleifforschungsprojekte behandeln daher Fragen des möglichen Missbrauchspotenzials, der Risiko-Nutzen-Verteilung oder rechtlicher und sozialwissenschaftlicher Auswirkungen. Eine ähnliche Tendenz lässt sich auch etwa in der Synthetischen Biologie feststellen, wo ebenfalls die sozialwissenschaftliche Begleitforschung breiten Raum einnimmt.

\section{Die österreichische Forschungslandschaft}

Einige österreichische Forschungseinrichtungen sind bereits in europäischen CT-Forschungsprojekten entweder als Partnerlnnen oder Koordinatorlnnen eingebunden. Hierbei handelt es sich sowohl um Universitäten (TU Wien, BO$\mathrm{KU}$ ) als auch um Unternehmen (FOTEC, Wittmann Battenfeld und Alicona Imaging). Joanneum Research und das sozialwissenschaftliche Forschungszentrum ICCR sind außerdem an EU-Begleitforschungsprojekten zu gesellschaftlichen Auswirkungen von $\mathrm{CT}$ beteiligt. Auf nationaler Ebene wurde hingegen noch kein spezifisches Forschungsprogramm für CT ausgeschrieben. Einige CT-relevante Aktivitäten und Projekte wurden allerdings in den vergangenen Jahren im Rahmen der BioNanoNet-Plattform abgewickelt.

\section{Sicht der österreichischen Forscherlnnen}

Im Großen und Ganzen wurde CT als ein Beispiel interdisziplinärer Forschung gesehen. Nur wenige Forscherlnnen können allerdings klar darlegen, warum CT zwar immer interdisziplinär, aber interdisziplinäre Forschung nicht immer CT ist. Die aus teils sehr unterschiedlichen Fächern kommenden Workshopteilnehmerlnnen begrüßten einheitlich die vermehrte fächerübergreifende Kooperation und hielten diese für die zukünftige Forschung und Technikentwicklung für sehr wichtig.

Von einigen Teilnehmerlnnen wurde angemerkt, dass eine Konvergenz zweier oder mehrerer Disziplinen oder Technologien in der Vergangenheit bereits wiederholt stattgefunden hat. An Beispielen wie Kybernetik oder Mechatronik könne man sehen, dass CT eigentlich schon mehrere Jahrzehnte alt sei. Trotzdem waren sich die Expertlnnen einig, dass momentan eine signifikante Veränderung in der Dynamik des kollaborativen Wissenserwerbs stattfindet. Es wurde mit einiger Sorge geäußert, dass Österreich, trotz einiger Vorzeigeaktivitäten, international den Anschluss verlieren könnte. Die Frage, ob es konkrete Hinweise für die besondere Aktualität der CT gäbe, blieb unbeantwortet. Vielmehr vermutet man in der CT einen neuen Wissenschafts- und Techno- 
logietrend, der gerade dabei sei, Fuß zu fassen, selbst aber noch kaum einen Fußabdruck hinterlassen habe.

Um CT-Forschung in Österreich zu fördern, wäre es laut ExpertInnen wichtig, F\&E-Programme möglichst offen und flexibel zu gestalten, die Gremien zur Evaluierung der Projekt- und Pub- likation interdisziplinär zu besetzen und die Möglichkeit einzuräumen, ohne Erfolgsgarantie zu forschen. Weiters müsse gewährleistet werden, dass Forschungsförderungsmittel neu aufgestellt und nicht aus bereits laufenden Programmen abgezogen werden.

André Gazsó

\section{Social Network Sites - ein Trend auch in der Wissenschaft?}

\begin{abstract}
Social Network Sites (SNS) gehören zweifellos zu den wichtigsten Internetphänomenen der letzten Jahre. Das zeigt sich etwa an den beeindruckenden Zahlen, mit denen Branchen-Primus Facebook aufwarten kann: $\mathbf{5 0 0}$ Millionen Nutzerlnnen sind hier angemeldet und der Marktwert des Unternehmens wurde kürzlich auf 41 Milliarden US-Dollar geschätzt' ${ }^{1}$. Daneben gibt es unzählige weitere Netzwerke, die (wie etwa StudiVz oder MySpace) ähnlich breit angelegt sind, oder eher auf berufliche (z. B. Xing) oder andere spezielle Bedürfnisse abzielen. Auch für WissenschafterInnen wurden und werden diverse Angebote entwickelt, die kaum zu überblicken sind2. Handelt es sich dabei lediglich um einen weiteren Internet-Hype, oder verbergen sich hier tatsächlich auch Potenziale für die Wissenschaftskommunikation?
\end{abstract}

Ein erster Blick auf die mannigfaltigen Funktionen der Dienste lässt ihre möglichen akademischen Einsatzgebiete erahnen: Kolleglnnen können hier über diverse Wege miteinander kommunizieren (z. B. Direktnachrichten, Chat, Foren, Microblogging), ihre bestehenden Netzwerke abbilden und neue aufbauen, Termine organisieren, in Gruppen kollaborieren u. v. m. Wissenschaftliche SNS wie ResearchGATE bieten darüber hinaus spezielle Funktionen für den akademischen Bedarf, wie beispielsweise die Suche nach ähnlichen Abstracts, das Erstellen von persönlichen Bibliografien, Zugriffsstatistiken für Texte usw. Mit Blick auf diese technischen Möglichkeiten scheinen SNS also durchaus auch für die wissenschaftliche Nutzung interessant zu sein.
Tatsächlich sind nicht wenige Wissenschafterlnnen bereits in SNS aktiv. Eine Studie offenbarte, dass immerhin $23 \%$ von über 2.300 befragten Wissenschafterlnnen deutscher Hochschulen SNS häufig oder sehr häufig nutzen. ${ }^{3}$ Es ist zu erwarten, dass diese Zahl zunimmt, denn beim akademischen Nachwuchs sind SNS erwartungsgemäß noch beliebter - hier nutzt bereits eine Mehrheit diese Dienste. ${ }^{4}$

Gleichzeitig ist zu fragen, wie sich die Nutzungspraxen der Wissenschafterlnnen in diesen Netzwerken konkret gestalten - worüber derartige Studien nur eingeschränkt Auskunft geben können. Verkehrt man in SNS, fällt schnell auf, dass viele Nutzerlnnen zwar über ein Profil verfügen, es sich dabei aber eher um eine "Me-too-Präsenz" handelt, d. h. dass sie kaum darüber hinaus aktiv sind. Besonders in allgemeinen SNS wie Facebook sind zudem häufig Nutzungsweisen beobachtbar, die eher privater Natur sind.

Die Probleme, die solche Praxen aus PrivacyPerspektive mit sich bringen, werden mittlerweile nicht nur in Fachkreisen, sondern auch in der Öffentlichkeit weitläufig diskutiert. Selbstredend harmonieren die hier aufeinander treffenden privaten und beruflichen Identitäten nicht immer miteinander. In diesem Zusammenhang stellt sich auch die Frage, wie effektiv SNS für wissenschaftliche Zwecke einsetzbar sind. Hier lässt sich ein Argument reaktivieren, das Internet-Kritikerlnnen bereits seit den frühen Tagen des Netzes immer wieder anbringen: Die Fülle der Informationen sind mitunter weniger hilfreich als problematisch. Gerade Neulinge haben häufig Schwierigkeiten, die vielen Funktionen und Informationen effektiv zu organi- 
sieren. Da die letztlich relevante (interne wie externe) Wissenschaftskommunikation noch immer primär über traditionelle Kanäle erfolgt, sind die Skepsis und das mangelnde Engagement vieler ForscherInnen gegenüber den neuen Plattformen verständlich. Gleichzeitig kann eine gezielte Nutzung der hier wachsenden Möglichkeiten aber gerade auch für Nachwuchswissenschafterlnnen, die noch nicht sehr in bestehende Strukturen und Netzwerke eingebettet sind, Chancen bieten.

So erscheint klar, dass SNS durchaus interessante Potenziale für den akademischen Bedarf aufweisen, während sie auch noch einige Hürden bewältigen müssen, bevor sie sich in diesem Bereich etablieren können. Das zeigt sich auch an den zahlreichen wissenschaftlichen SNS, die in den letzten Jahren entwickelt wurden. Sie verweisen einerseits auf den potenziellen Bedarf, andererseits aber auch auf noch nicht erfolgte Kristallisierungs- und Reifungsprozesse. Denn Monopolisierungstendenzen, wie sie sich bei allgemeinen SNS in Gestalt von
Facebook andeuten, sind in diesem Fall prinzipiell funktional, schließlich steigt der Nutzen eines Netzwerks mit der Anzahl seiner aktiven Mitglieder. Dies gilt auch für wissenschaftliche SNS, wenn auch in kleinerem Rahmen. Gleichzeitig führen derartige Quasi-Monopole natürlich zu Abhängigkeiten, die - je nach hintergründiger Geschäftspolitik - durchaus problematisch sein können. Das zeigt sich u. a. am Beispiel Facebook, das immer wieder Negativschlagzeilen mit Datenschutzfragen macht.

Diese Entwicklungen sind Gegenstand eines Berichts, der demnächst im Rahmen des Projekts Interactive Science erscheint:

epub.oeaw.ac.at/ita/ita-projektberichte/ d2-2a52-5.pdf.

1 www.businessweek.com/news/2010-11-15/facebook-passesebay-in-value-becoming-no-3-u-s-web-company.html.

2 Einige davon werden hier vorgestellt: www.geozon.info/ 2010/03/08/wissenschaftliche-netzwerke-teil-1-4/.

3 eleed.campussource.de/archive/5/1842/.

4 https://hisbus.his.de/hisbus/docs/hisbus21.pdf.

René König

\section{Dekonstruktion und (Ver-)Lernen in der Softwareentwicklung}

\begin{abstract}
Im wissenschaftlichen Diskurs wird von der wechselseitigen Gestaltung von technologischem und gesellschaftlichem Wandel ausgegangen. In diesem Sinne konstruieren Technologien soziale Verhältnisse sowie Alltags- und Arbeitspraktiken mit und umgekehrt werden in ihrer Entwicklung auch gesellschaftliche Diskurse wirksam. Constructive Technology Assessement (CTA) zielt in diesem Zusammenhang darauf ab, soziale Auswirkungen technologischer Innovationen vorwegzunehmen und bereits im Rahmen ihrer Entwicklung Reflexivität und gesellschaftliches Lernen zu verankern.
\end{abstract}

Softwareentwicklung findet in sozialen multidimensionalen Aushandlungsprozessen statt. Dabei werden alltägliche Arbeitspraktiken in Entwicklungsteams vor dem Hintergrund gesellschaftlicher Diskurse verhandelt. So werden Designentscheidungen u. a. durch implizite und damit unreflektierte Annahmen über zukünftige Nutzungskontexte getroffen - etwa auf Basis soziodemografischer Aspekte wie Alter, Geschlecht, Behinderung und sozialer oder ethnischer Hintergrund der Nutzerlnnen. Diese Aushandlungsprozesse sind zudem durch formale Organisationsstrukturen wie auch durch informelle Wissenshierarchien geprägt und zeichnen sich in der Regel durch ein stark technikzentriertes professionelles Selbstverständnis der Entwicklerlnnen aus. Soziale Dimensionen und normative Implikationen werden daher weitgehend ausgeblendet. Sie bleiben unsichtbar und können somit nicht verhandelt werden. Stattdessen wird in den verschiedenen Phasen des Systemdesigns oft unbewusst auf eingefahrene Lösungswege zurückgegriffen, die sich ungünstig auf werterelevante Produktqualitäten auswirken.

Vor diesem Hintergrund stellen wir in zwei Artikeln $2010^{1}$ einen Softwareentwicklungsansatz vor, der Dekonstruktion und (Ver-)Lernen als reflexive Praktiken in den Entwicklungsprozess in- 
tegriert: „Deconstructive Design" macht sichtbar, wie informelle Hierarchien und hegemoniale gesellschaftliche Diskurse in konkreten Softwareentwicklungen wirksam werden. Entwicklungsprozesse werden damit als kollektive Praktiken begreifbar, die gesellschaftlich Bedeutungen (re-)produzieren. Entwicklungsentscheidungen werden häufig auf Basis evidenter Kausalitäten getroffen. Als Methode zielt Dekonstruktion daher darauf ab, die Konstruiertheit und Normativität dieser scheinbar "natürlichen" Verknüpfungen deutlich zu machen. In diesem Sinne können einschränkende Automatismen wie etwa Stereotypisierungen verlernt werden.

Die dekonstruktivistische Methodologie wird mit praxis-basierten Konzepten von situiertem, $d$. h. in den Arbeitsprozess eingebettetem Lernen verknüpft. „Deconstructive Design" stellt ein Verfahren zur Verfügung, das Teams dabei unterstützt, ihre eigenen Arbeitspraktiken kritisch zu betrachten und eine Auseinandersetzung mit der sozialen Bedeutung dieser Praktiken unmittelbar in den Entwicklungsprozess einzubeziehen. Auf diese Weise können sich die Entwicklungsteams nachhaltig Reflexionskompetenzen aneignen.
Anknüpfend an die Tradition reflexiver und werte-sensibler Entwicklungsansätze wird damit ein Potential für werte-basierte Intervention in die Gestaltung von Informations- und Kommunikationstechnologien auf der Ebene kollektiver Arbeitspraktiken verortet. Im Unterschied zu diesen Ansätzen sollen im Rahmen des dekonstruktivistischen Zugangs allerdings die Werte selbst hinterfragt werden, auf Basis derer informelle Hierarchien und diskursive Hegemonien normative Wirkmacht erlangen.

Einem solchem Vorhaben liegt ein Verständnis von Software als sozial verhandelte Konstruktionen zugrunde. Werden die gesellschaftliche Relevanz und die Wertegeladenheit dieser Konstruktionen sichtbar gemacht, können auch scheinbar objektive und gesellschaftspolitisch neutrale Konzepte und Theorien angewandter Informatik in Frage gestellt werden. Reflexive Praxis eignet sich auf diese Weise auch dazu, Grundparadigmen positivistischer Ansätze der Informatik kritisch zu betrachten.

In: Vallverdú, J. (ed.), Thinking Machines and the Philosophy of Computer Science, Hershey/New York, $175-$ 192, und in: Poiesis \& Praxis 7(1-2), 87-98.

Doris Allhutter, Roswitha Hofmann

\section{Technologiespezifische Perspektiven auf digitale Pornografie}

\begin{abstract}
Pornografie ist nicht zuletzt durch das Internet mittlerweile zum massenkulturellen Phänomen geworden. Auf der Konferenz „Pornografisierung von Gesellschaft?!", die vom 28.-30.10. an der Fachhochschule Köln stattfand, wurde dieses Phänomen in seinen vielfältigen Facetten beleuchtet. Diskutiert wurde seine Bedeutung für die Kommunikationskultur und Lebenswelt von Jugendlichen und Erwachsenen. Das ITA war mit einer Keynote vertreten.
\end{abstract}

Ziel ist es, „Jugendliche zu einem selbstbestimmten und verantwortetem Umgang mit Pornografie zu befähigen", brachte Mitveranstalterin Angela Tillmann, Institut für Medienforschung der FH Köln, den Tenor der Konferenz auf den Punkt. Eine Veränderung sexueller Stan- dards bei Jugendlichen sei nicht festzustellen, so der Sozialpädagoge Uwe Sielert. Verändert habe sich aber die Qualität und Quantität, in denen sexuelle Themen kommuniziert werden. Gerade diese scheinbare Selbst-Ermächtigung qua Körper und qua selbstbestimmter Sexualität, die medial ausgeschlachtet und bei den Zuseherlnnen angeregt wird, sei aber kritisch, erläuterte die Soziologin Paula-Irene Villa. Junge Frauen versuchen sich durch Sexualisierung als aktives, selbstverantwortliches Subjekt zu setzen und präsentieren sich beispielsweise auch dementsprechend im Internet. Im durchökonomisierten Kapitalismus, so Villas These, sei dies ein Versuch, dem Status des passiven "Opfers" zu entkommen. Ein „Empowerment"Potenzial, in Hinblick auf eine Veränderung unserer Gesellschaft in Richtung Geschlechter- 
demokratie, beinhalte diese Selbstinszenierung aber nicht. In Bezug auf Jugendliche und auch auf Erwachsene bleiben in diesem Sinne weiterhin geschlechterrelevante Mechanismen der Inszenierung von Pornografie zentral.

Die bereits in den 1980er Jahren intensiv geführte Debatte über Pornografie hat insbesondere mit dem pornografischen Massenangebot im Internet wieder an wissenschaftlicher Bedeutung gewonnen. Mehr als die möglichen Auswirkungen des Porno-Konsums steht nun die Frage im Mittelpunkt, was die Inhalte dieser medialen Repräsentationen über Geschlechterverhältnisse und globale ökonomische Machtstrukturen aussagen. Die spezifische Auseinandersetzung mit dem Einsatz von Informationsund Kommunikationstechnologien für pornografische Zwecke konzentriert sich - beispielsweise im Rahmen der Computerethik - weitgehend darauf zu erforschen, wie das Internet für die Verbreitung pornografischer Materialien verwendet wird. Mit der Nutzung digitaler Technologien veränderte sich allerdings nicht nur die Menge an Pornografie und ihre Verbreitungswege, stellte Doris Allhutter (ITA) im Rahmen ihres Keynote-Vortrags fest. Es entstand auch eine Vielfalt an neven Formaten und pornografischen Computeranwendungen wie etwa Porno-Suchmaschinen, computer-generierte 3D-Animatonen und Computerspiele. Eine technologie- bzw. medienspezifische Analyse muss in diesem Sinne auch die Ver- änderungen des Genres sowie veränderte technologische Herstellungs- und Aneignungsprozesse in den Blick nehmen. ${ }^{1}$

So zeigt sich etwa in der Entwicklung digitaler 3D-Animationen, wie Ideologien menschlicher Verschiedenheit dazu eingesetzt werden, pornografisch inszenierte Körper, Bewegungen und Szenarien zu simulieren. Die Übersetzung dieser Ideologien in Computercodes dient dazu, computergenerierte Simulationen als „menschlich" glaubhaft zu machen und verfestigt in ihrer anatomisch-technologischen Akribie sexistische, heteronormative und rassistische Vorstellungen. Schon vor der Verbreitung des Internet wurde die Aneignung medialer Inhalte nicht lediglich als individuelles Konsumieren verstanden und in ihren möglichen Auswirkungen auf Individuen untersucht. Gerade die Vernetzung und die Möglichkeiten der Nutzerlnnen, sich aktiv an der Herstellung oder Verbreitung von Inhalten zu beteiligen, machten aber wieder deutlicher, dass Medienrezeption ein kollektiver, tätiger Aneignungsprozess ist. Im Zusammenhang mit digitaler Pornografie involvieren sich Entwicklerlnnen und Userlnnen durch ein interaktives Zusammenbasteln von pornografischen Imaginationen, die großteils auf den genannten Ideologien basieren.

1 Allhutter, D. 2009, Dispositive digitaler Pornografie. Zur Verflechtung von Ethik, Technologie und EU-Internetpolitik, Frankfurt/New York: Campus.

Doris Allhutter

\section{Interdisziplinarität und Technowissenschaft}

\section{Die Diskussion um Interdisziplinarität in der Forschung scheint nicht abzurei- Ben. Derzeit erlebt der Begriff der In- terdisziplinarität eine neue Konjunktur, zu der neue Entwicklungen in den Tech- nowissenschaften beitragen.}

Bereits seit den 1970er Jahren wird unter diesem Schlagwort diskutiert, wie universitäre Ausbildung und Forschung der Bearbeitung konkreter gesellschaftlicher Problemstellungen gerecht werden kann. Das schließt Fragen des Umweltschutzes, der Nachhaltigen Entwicklung und der Behandlung schwerer Krankheiten und Epidemien ein. Zugleich bemühen sich Wissen-
schaftsforscherInnen um eine detaillierte Charakterisierung und ein Verständnis interdisziplinärer Forschung als Sonderform von Wissenschaft. Und nicht zuletzt geht es auch darum, Qualitätssicherungs- und Fördermechanismen für diesen Sonderweg bereitzustellen. Eine Aufgabe, der sich auch der FWF, Österreichs zentrale öffentliche Förderinstitution für Grundlagenforschung, bis heute nicht immer gewachsen sieht. "Es bleibt eine Herausforderung", so steht in der Oktober-Ausgabe des FWF-Magazins zu lesen, „ob und wie sich interdisziplinöre Projekte zielgerecht identifizieren lassen und wie man dementsprechend die Entscheidungsverfahren anpassen kann." Es könne da- 
her durchaus so sein, dass interdisziplinäre Projekte nur schwer zu Fördergeldern kämen, obwohl sie doch durchaus erwünscht seien.

Während aber einerseits die Reihe gesellschaftlicher Problemstellungen nicht abzureißen scheint, die dem Ruf nach problem-orientierter interdisziplinärer Forschung zu Grunde liegt, hat sich in den Wissenschaften auch eine weitere Grundlage für Kooperation zwischen den Disziplinen herausgebildet. Es ist dies der Versuch, naturwissenschaftliche Erkenntnisse, Techniken und Objekte technologisch einzusetzen, oder umgekehrt, den technologischen Ansatz in die naturwissenschaftliche Grundlagenforschung zu integrieren. Zwar versprechen die darauf basierenden Technologien auch Lösungen für gesellschaftliche Problemen zu liefern, vorerst geht es aber vor allem um die Konstruktion never Systeme und die Entwicklung neuere Machbarkeiten. Besonders neu ist diese Entwicklung für die Lebenswissenschaften. Prominente Beispiele finden sich in der Systembiologie, der synthetischen Biologie, der Nanobiotechnologie und den Neurowissenschaften - also jenen Feldern, die mitunter unter dem Schlagwort "Technowissenschaff" subsumiert werden. Gerade diese technowissenschaftlichen Felder sind es auch, in denen sich im Rahmen von Begleitforschung Kooperationen mit den Sozial- und Geisteswissenschaften etablieren und weiter entwickeln.

Vor diesem Hintergrund, der erst nach und nach auch von jenen ExpertInnen wahrgenommen wird, die sich schon länger mit Interdisziplinarität befassen, erscheinen bereits bekannte Spielformen, Möglichkeiten und Schwierigkeiten von Interdisziplinarität in einem neuen Licht. Während das innovative Potenzial wissenschaft- lichen Austausches über Disziplinengrenzen hinweg unumstritten ist, gelten ,alte' Probleme wie jenes tragfähiger interdisziplinärer Kommunikation, Institutionalisierung und Qualitätssicherung nach wie vor als ungelöst.

Dass der technowissenschaftliche Trend zu Interdisziplinarität inzwischen zunehmend Aufmerksamkeit erregt und damit für eine neverliche Konjunktur des Begriffes sorgt, spiegelt sich in aktuellen Konferenzen, Workshops, Publikationen und Diskussionen wieder. So richtete die Universität Exeter (Großbritannien) eine Tagung zu "Frontiers of multidisciplinary research" aus, in der es um die Erschließung never, multidisziplinärer Ansätze zwischen Mathematik, Ingenieurwissenschaften und Biologie in der Systembiologie ging. Das transnationale Netzwerk "Philosophy of/as Interdisciplinarity" widmete sich in einem Workshop der philosophischen Bearbeitung von Interdisziplinarität, wie auch der Weiterentwicklung methodischer Ansätze, in denen PhilosophInnen und Technowissenschaftlerlnnen zusammenarbeiten. Und, last but not least, legte Jutta Weber kürzlich einen Band zu "Interdisziplinierung?" vor, der explizit auf die Situation in den Technowissenschaften eingeht.

Zum Nachlesen:

www.exeter.ac.uk/research/excellence/ keythemes/systemsbiology/frontiers/; www.pin-net.gatech.edu/i nternational_workshop2010.php.

Weber, J. (Hg.), 2010, Interdisziplinierung? Zum Wissenstransfer zwischen den Geistes-, Sozial- und Technowissenschaften, Bielefeld: transcript.

Karen Kastenhofer

\section{Technology Governance: Wie kann TA dazu effektiv beitragen?}

\author{
Der kürzlich erschienene Tagungsband \\ zur NTA3-TA'08 mit dem Titel „Techno- \\ logy Governance" diskutiert facetten- \\ reich die möglichen Beiträge der TA zum \\ gesellschaftlich-politischen Umgang mit \\ Technologieentwicklung.
}

Der gesellschaftliche Wandel legt aus mehreren Gründen eine kontinuierliche Auseinandersetzung mit Voraussetzungen und Reichweite der TA nahe, wenn diese einen wirksamen Beitrag zur gesellschaftlichen Entwicklung leisten soll. Dazu zählt zuvorderst der Wandel der Innovationssysteme und Grundlagen der Tech- 
nikentwicklung. Neben einer zunehmenden Verschränkung von Politik, Wirtschaft und Wissenschaft gehören dazu etwa die enorme Beschleunigung von Innovationsprozessen (bzw. die Verkürzung von Innovationszyklen), die Ausweitung des Spektrums an Akteurlnnen und strategischen Orten des Innovationsgeschehens einschließlich never Formen netzwerkartiger Kooperationsbeziehungen, ferner die Auflösung linearer Forschungs- und Entwicklungsprozesse, das Verschwimmen der Grenzen zwischen Grundlagen und angewandter Forschung (bzw. technischer Entwicklung) sowie nicht zuletzt auch ein gestiegenes Interesse der Öffentlichkeit an Technologieentscheidungen. Diese Veränderungen werden oft als steigende Komplexität und Kontrollverlust interpretiert.

Für die TA stellt sich daher die Frage, wie sie sich auf neue Herausforderungen einzustellen vermag, um eine Einflussnahme auf die technische Entwicklung zur Förderung des Gemeinwohls möglichst effektiv zu unterstützen. Zugleich geht es dabei um eine Stärkung von Konzeption und Praxis der TA, die den Vorwurf politischer Wirkungslosigkeit ebenso zu widerlegen in der Lage ist wie jenen eines naiven Steuerungsoptimismus. Einiges spricht dafür, eine solche Reflexion aus einer Governance-Perspektive zu unternehmen. Schließlich hat sich Governance als „interdisziplinärer Brückenbegriff" etabliert und seine analytische Fruchtbarkeit mit der Anwendung in zahlreichen Kontexten unter Beweis gestellt.

Im Begriff "Technology Governance" spiegelt sich ein geändertes Verständnis der Steverbarkeit technischen Wandels und der Regelungsstruktu- ren, in denen öffentliche und private Akteurlnnen zusammenwirken. Als Reflexions- und Wissensressource im Governance-Prozess versucht TA Chancen und Risiken never Technologien auszuloten sowie zum größtmöglichen gesellschaftlichen Nutzen auf Entwicklung und Anwendung einzuwirken. Die Zentralität technischer Innovation im globalen Wettbewerb verstärkt den Bedarf an Folgenwissen und damit an TA. Effektive Beiträge zur Governance technologischer Entwicklungen hängen allerdings von der geeigneten Ausrichtung und Ausstattung der TA ab.

Der bei edition sigma erschienene Band "Technology Governance: Der Beitrag der Technikfolgenabschätzung" ${ }^{11}$ bietet eine Reihe von Ansätzen, die sich mit Anforderungen an die TA und deren Wirkungsseite in unterschiedlichen Anwendungskontexten aus einer GovernancePerspektive befassen. Ausgehend von Grundsatzfragen des Einsatzes von TA für Technology Governance und einer Betrachtung verschiedener TA-Konzepte im Lichte von Governance untersuchen weitere Kapitel des Bandes mögliche Funktionen und Fragen des Timings von TA im Governance-Prozess sowie eine Analyse von Mikrostrukturen der Innovationsgestaltung. Der Bogen thematisierter Technisierungsprozesse spannt sich von der Bio- und Nano-, über die Informations- und Energietechnologie bis zu Human Enhancement-Technologien und schließt auch Beiträge mit ein, deren Fokus auf nachhaltiger Entwicklung liegt.

Aichholzer, G., Bora, A., Bröchler, S., Decker, M., Latzer, M. (Hrsg.): Technology Governance. Der Beitrag der Technikfolgenabschätzung, Berlin: edition sigma, 2010.

Georg Aichholzer

\section{Geschichte der Nanotechnologie - Bericht von der 2. S.NET-Konferenz}

\author{
Vor genau einem Jahr berichteten wir \\ von der 1. Konferenz der "Society for the \\ Study of Nanoscience and Emerging \\ Technologies" (S.NET). Heuer fand die \\ 2. Konferenz vom 29.9-2.10. in Darm- \\ stadt statt. Mit 130 TeilnehmerInnen und \\ etwa 100 Beiträgen übertraf sie noch \\ ihre Vorgängerin in Seattle. Auch das \\ ITA war mit einer Session vertreten.
}

Entsprechend dem Ziel von S.NET - den intellektuellen Austausch von Wissenschafterlnnen aus dem Bereich der Reflexionswissenschaften über die gesellschaftliche Bedeutung der $\mathrm{Na}$ notechnologie (NT) zu fördern - standen auch auf dieser Konferenz nicht-technische Aspekte des Phänomens NT im Mittelpunkt. Ziel der Gesellschaft ist es darüber hinaus, den transatlantischen Dialog auf diesem Gebiet voranzutrei- 
ben. Knapp ein Viertel der Teilnehmerlnnen kamen aus den USA, 2/3 kamen aus Europa, ca. 1/3 der Teilnehmerlnnen kam alleine aus Deutschland. Interessant war, dass Frankreich, sonst eher dafür bekannt im Bereich der TA unterrepräsentiert zu sein, ca. $10 \%$ stellte.

Auffällig war, dass Arie Rip (Universität Twente, S.NET-Präsident) schon in der Einleitung auf die mögliche Transformation der NT als Phänomen der Wissenschaftsorganisation hinwies. Er beobachte ein Abklingen des Hypes, was mit einer Konsolidierung und Spezialisierung innerhalb der NT einhergehe. Diese Stimmung war durchaus kennzeichnend für die Konferenz. Daher werden hier im Weiteren zwei Plenarvorträge kurz vorgestellt, die beide einen Rückblick auf die letzten zehn Jahre der NT gaben.

Richard Jones (Universität Sheffield) diskutierte in seinem Vortrag die wesentlichsten Aspekte, die zur Formierung der NT beigetragen hatten und die sie jetzt kennzeichnen. Er rekonstruierte die NT als eine Re-Konfiguration vieler schon vorher bestehender wissenschaftlicher Aktivitäten, die sich unter dem Begriff NT zusammengefunden haben. Am Anfang stehen vor allem außerwissenschaftliche Impulse wie die Formulierung von zielgeleiteten und innovationsorientierten Forschungsprogrammen oder das Aufbrechen von großen wirtschaftlichen Einheiten der Versuch eines schnelleren Technologietransfers mittels kleiner ausgelagerten Unternehmen.

Der Vortrag von Jones hatte die verschiedenen Bereiche der NT (Feinmechanik, Mikroelektronik, molekulare Elektronik, organische Elektronik, Clusterchemie, Oberflächenphysik, Materialforschung, molekulare Biophysik etc.) so plausibel ineinander verwoben, dass es eher erstaunlich schien, dass die Idee, all dies mittels einer Erzählung zusammenzuführen, erst so spät aufgekommen ist. Auf die Frage, warum dies so sei, antwortete Jones, dass es zunächst einer Abkehr vom „linearen Modell“ technischer Innovationen bedurfte. Damit wurde die Zuversicht brüchig, dass Grundlagenforschung automatisch zu technologischem Fortschritt führe.
Armin Grunwald (ITAS, Karlsruhe) fragte in seinem Vortrag nach der Wirkung, die die Debatte um die NT auf deren Entwicklung hatte. Er unterschied hierbei einen direkten Einfluss im Sinne von Technikgestaltung und einen indirekten Einfluss, bei dem es eher darum ging, Rahmenbedingungen zu beeinflussen und Ziele mit zu formulieren. Sein Fazit lautete, dass die Debatte weniger direkten Einfluss hatte, er aber sehr wohl einen indirekten Einfluss beobachten konnte. Sein Vortrag löste eine rege Diskussion aus, welche die sehr verschiedenen Positionen im Publikum verdeutlichte. Auf der einen Seite wurde kritisiert, dass die analytische Perspektive fraglich sei, da ihre Voraussetzungen und Verortung im sozio-technischen System nicht mitreflektiert würden (Ulrike Felt). Auf der anderen Seite, sozusagen antagonistisch zur Kritik von Felt, wurde von Rene von Schomberg hervorgehoben, dass die Debatte um NT ganz wesentlich die Gestaltung des 6. und 7. Forschungsrahmenprogramms der EU beeinflusst habe und man daher eher von einer direkten Einflussnahme sprechen sollte.

Die gesamte Tagung war durch sehr unterschiedliche Themen und Zugangsweisen zur Analyse der NT gekennzeichnet. Das Spektrum reichte von ethischen und politikwissenschaftlichen Fragen, insbesondere zur Verantwortung, über Analysen epistemischer und disziplinärer Veränderungen in der Forschungspraxis bis hin zu Untersuchungen über Visualisierungen der NT und deren literarischen und cineastischen Bezügen.

Das ITA beteiligte sich im Rahmen des NanoTrust-Projekts an der Organisation einer Session über den Vergleich der Nano-Politiken von Deutschland, Österreich und der Schweiz. Im Rahmen dieses Kooperationsprojektes des ITA mit deutschen und schweizer Wissenschafterlnnen werden Themen, Akteurlnnen, partizipative Veranstaltungen, Regulierungsaktivitäten sowie die mediale Berichterstattung in den verschiedenen Ländern untersucht und verglichen. Auf der S.NET wurden erste Ergebnisse des Projekts vorgestellt und diskutiert.

Informationen zum S.NET sowie zur Konferenz: www.thesnet.net. 


\section{Technikfolgenabschätzung international: EPTA-Jahrestagung 2010}

\begin{abstract}
Das Netzwerk der europäischen parlamentarischen TA-Einrichtungen (European Parliamentary Technology Assessment - EPTA) traf sich hever von 2.-3.11. zur jährlichen Sitzung des Council und zur Jahrestagung in Kopenhagen. Der EPTA-Council verabschiedete unter anderem einen Kodex zur Zusammenarbeit unter den Mitgliedern, kürte die nächste Präsidentschaft und nahm eine erste außereuropäische TA-Einrichtung als assoziiertes Mitglied auf. Die Tagung im dänischen Parlament war dem Thema Nachhaltiger Verkehr gewidmet.
\end{abstract}

Die EPTA-Mitglieder treffen einander nicht nur regelmäßig zum Gedankenaustausch, etliche von ihnen arbeite(te)n auch in EU-Projekten zusammen (201) startet wieder ein gemeinsames Project: PACITA - Parliaments and Citizens in Technology Assessment). Daneben gab und gibt es auch gemeinsame EPTA-Projekte, etwa zum Thema Privacy (siehe ITA-News Dez. 2006, S. 2) oder zur Gentechnik in der Landwirtschaft (siehe ITA-News Juni 2009, S. 10). Wie diese Projekte zustande kommen und intern organisiert werden, aber auch welche Regeln für Mitgliedschaften, EPTA-Gremien und Entscheidungsfindungen bestehen, wurde nunmehr vom EPTA-Council in Form einer Richtlinie niedergelegt, die im Wesentlichen die bisherige Praxis festhält. Danach können EPTA-Projekte von mindestens drei Mitgliedern gestartet werden. Die Projektkosten werden von den Partnern selbst getragen. Jüngstes Beispiel ist der in Vorbereitung zur Jahreskonferenz gemeinsam erstellte Bericht "Routes to sustainable transport. An overview of status and policy strategies for sustainable transport in Europe" 1 , der einen Überblick über 13 Länder plus der EU ermöglicht; auch Österreich ist mit einem Beitrag vertreten.

In der diesjährigen Council-Sitzung wurde weiters beschlossen, das Government Accountability Office $(G A O)^{2}$ des US-amerikanischen Kongresses als assoziiertes Mitglied aufzunehmen. Dessen Abteilung "Center for Science, Technology, and Engineering (CSTE)" erstellt seit 2007 im Auftrag des US-Parlaments unter anderem auch TA-Studien. Bisherige Projekte wid- meten sich etwa der Biometrie, der Cyberkriminalität und Sicherheitsfragen beim Personenschienenverkehr. Aktuell beschäftigt sich GAO u. a. mit Ölunfällen und Geo- bzw. Klima-Engineering. Damit wurde zum ersten Mal eine außereuropäische Institution EPTA-Mitglied.

EPTA verfolgt auch intensiv die Entwicklung im Bereich TA in Japan und wird umgekehrt von VertreterInnen der dort entstehenden TA-Einrichtungen beobachtet. Diesmal stellte sich die Einrichtung "Innovation and Institutionalization of Technology Assessment (I2TA)" vor. ${ }^{3}$ Dabei handelt es sich um ein Projekt zur Etablierung von TA in Japan, das vom Research Institute of Science and Technology for Society (RISTEX) der Japan Science and Technology Agency (JST) getragen wird. Aktuell widmet sich I2TA dem Bereich Nanotechnologien, mit Schwerpunkten auf Lebensmittel, Energie und Medizin.

Ebenfalls festgelegt wurde die kommende EPTA-Präsidentschaft: 2011 wird das Büro für Technikfolgenabschätzung (TAB) beim Deutschen Bundestag nach den dänischen Kolleglnnen den Vorsitz übernehmen. Ende September wurde in Berlin das zwanzigiährige Bestehen des TAB im Bundestag feierlich begangen. Die konkrete institutionelle Verankerung des $T A B$ ist international einzigartig und beispielgebend. Dies wurde bei der Festveranstaltung wiederum eindrücklich sowohl von Seiten der Bundestagsabgeordneten als auch von Seiten der Wissenschaft und der TA-Praxis bestätigt.

Die EPTA-Jahreskonferenz war heuer den möglichen Wegen zu einem nachhaltigen Verkehrswesen gewidmet. Ausgangspunkt war der oben erwähnte gemeinsame Bericht der EPTA-Mitglieder. Die fünfzehn Vorträge waren auf fünf Programmblöcke aufgeteilt: (1) Herausforderungen, Perspektiven, aktuelle Entwicklungen und Politiken, (2) Energieverbrauch, (3) neue technologische Lösung, (4) grenzüberschreitender Verkehr innerhalb Europas und (5) alternative Politikansätze. Österreich war mit einem Vortrag von Willy Raimund (Energieagentur) im Bereich (4) vertreten. In Anlehnung an das System der Hearings im dänischen Parlament wurden die Vorträge von wechselnden Panels kom- 
mentiert, die hauptsächlich aus Abgeordneten mehrerer europäischer Länder zusammengesetz† waren. Die Vorträge und Diskussionen sind im Internet zum Download und zum Nachhören dokumentiert. ${ }^{4}$
1 www.tekno.dk/EPTA/documents/2010/ p10_EPTA_routes_to_sustainable_transport.pdf.

2 www.gao.gov.

3 i2ta.org/english/english.html.

4 www.tekno.dk/EPTA/news.php?article=27.

\section{Risikobewertung und Risikomanagement der Nanotechnologien}

\begin{abstract}
Am 23.9. fand die 4. NanoTrust-Herbsttagung statt. Während viele Aktivitäten bezüglich des Umgangs mit Risiken der Nanotechnologien sich darauf konzentrieren, Wissenslücken aufzuspüren bzw. diese zu schließen, war es Ziel dieser Tagung, Handlungsoptionen zu diskutieren, die sich der Situation des unzureichenden Wissens stellen: Wie geht man mit Risiken um, die man nicht genau kennt?
\end{abstract}

In den letzten Jahren hat sich der Diskurs über Risiken der Nanotechnologien auf Gesundheits- und Umweltrisiken von Nanopartikeln verengt. Zunehmend Einigkeit herrscht darüber, welche bestehenden Wissenslücken einer soliden Risikobewertung derzeit im Wege stehen. Forschungsprogramme werden formuliert und eine Reihe von Forschungsprojekten werden bereits durchgeführt, um diese Wissenslücken zu schließen. Ergebnisse als Basis für eine solide Risikobewertung sind aber erst in einigen Jahren zu erwarten. Nichtsdestotrotz müssen jetzt Entscheidungen über angemessene Vorsichtsmaßnahmen getroffen werden. Diese Problematik war Thema der diesjährigen NanoTrust-Herbsttagung.

Die Tagung näherte sich dem Thema von zwei Seiten. Einerseits wurde ein Beispiel aus einem anderen technologischen Feld (Mobilfunkstrahlen) betrachtet, andererseits berichteten PraktikerInnen aus Industrie, Verwaltung und der Versicherungswirtschaft, wie sie konkret mit der oben beschriebenen Situation umgehen.

In ihrem Einführungsvortrag gab die US-amerikanische Risikoforscherin Jo Anne Shatkin (Conservation Law Foundation) zunächst einen Überblick über das bestehende Wissen und die Wissenslücken sowie über die derzeitigen Risikomanagementaktivitäten. Anschließend stellte sie den Ansatz der Lebenszyklusanalyse (Life Cycle Analysis) vor. Im Rahmen des Risikomanagements von Nanomaterialien eignet sich dieses Instrument insbesondere zur Entwicklung von möglichen Expositionsszenarien und zur Priorisierung weiterer Risiko- und toxikologischer Forschung.

Am Beispiel der elektromagnetischen Strahlung diskutierte Peter Wiedemann (KIT, Karlsruhe) prinzipielle Probleme des Risikomanagements bei unsicherer Datenlage. Er verdeutlichte den Unterschied zwischen der Identifizierung von "tatsächlichen" Gefahren, von der das evidenzbasierte Risk Assessment ausgeht, und dem Ansatz der Risikosoziologie. Bei letzterer ist Risiko zunächst eine soziale Konstruktion, deren wesentliches Element die Risikowahrnehmung, also die subjektive Gefährdungszuschreibung zu denkbaren Ereignissen ist. Am Beispiel des Mobilfunks konnte Wiedemann zeigen, dass die Befriedung dieser Kontroverse nicht mit wissenschaftlichen Mitteln zu erreichen ist. Eine besonders interessante Beobachtung war, dass offen kommunizierte wissenschaftliche Unsicherheit von der Bevölkerung als mangelnde Qualität der Forschungsstudien gewertet wird.

Anschließend folgten konkrete Beispiele des Risikomanagements von Nanomaterialien. Karin Wiench (BASF) referierte kurz die wesentlichen Aspekte der evidenzbasierten Risikobewertung und konzentrierte sich dann auf die detaillierte Darstellung der Ergebnisse von toxikologischen Studien, an denen die BASF beteiligt war.

Markus Berges (Institut für Arbeitsschutz der deutschen gesetzlichen Unfallversicherungen, IFA) stellte Ergebnisse von Expositionsmessun- 
gen vor. Sein Vortrag verdeutlichte die enormen praktischen Schwierigkeiten bei der Bestimmung der Partikelkonzentrationen in der Atemluft. Insbesondere wird in der Regel nur die Anzahl der Partikel gemessen, nicht aber deren Größe und schon gar nicht deren chemische Zusammensetzung. Ein weiteres gewichtiges Problem ist die hohe Hintergrundkonzentration von nicht synthetisch hergestellten Partikeln, die die Interpretation der Messungen erschweren. Im zweiten Teil des Vortrages stellte Berges die Prinzipien des vom IFA entwickelten Benchmark-Ansatzes vor, der klare Ziele und Handlungsanweisungen beinhaltet' .

Astrid Epp (Bundesinstitut für Risikobewertung, BfR) referierte zunächst die Aufgaben und Arbeitsgebiete des BfR, deren Hauptziel der gesundheitlichen Verbraucherlnnenschutz ist. Das Institut ist schon lange im Bereich der Risikobewertung und Risikokommunikation der $\mathrm{Na}$ notechnologien aktiv. Aus Sicht des Verbraucherschutzes ist die mangelnde Information über die Verwendung von Nanomaterialien in Konsumprodukten eines der Hauptprobleme. Vor dem Hintergrund der Erfahrungen mit giftigen Chemikalien wie Blei oder polychlorierten Bephenylen (PCB) stellten jedoch für Epp die Nanopartikel weder eine neve Risikokategorie dar noch seien die Probleme nicht lösbar. So verwies sie in ihrem Vortrag auf die Kriterien für eine vorläufige Risikoabschätzung, die im Rahmen des NanoDialog-Projektes ausgearbeitet und von der deutschen NanoKommission veröffentlicht wurden².

Abschließend gab Thomas Jakl (BMLFUW) als Vorsitzender des Verwaltungsrats der Europäischen Chemikalienagentur (ECHA) einen Überblick über die europäische Chemikalien-Regulierung (REACH) sowie die laufenden $\mathrm{Be}$ mühungen, Nanomaterialien in diesem Regelwerk zu berücksichtigen. Obwohl REACH zunächst keine spezifischen Regeln für Nanoma- terialien enthält, müssen prinzipiell alle sicherheitsrelevanten Informationen zu einem Material angegeben werden, $d$. h. auch Eigenschaften, die sich erst ab einer bestimmten GröBe zeigen. In der Praxis erweist sich aber schon die Identifikation von Nanomaterialien als schwierig. Daher befasst sich die ECHA neben der Vereinheitlichung der Definition von Nanomaterialien mit der Ausarbeitung eines Leitfadens zur Information der betroffenen Unternehmen.

Betrachtet man das für NanoTrust wichtige sekundäre Ziel der Tagung, nämlich einen Rahmen zu schaffen, der es erlaubt, über die Reflexion der Risiken der Nanotechnologie hinauszugehen und auch praktische Erfahrungen auszutauschen sowie konkrete Kriterien und Maßnahmen für die Risikogovernance der $\mathrm{Na}$ notechnologie zu erarbeiten, so kann die Tagung als voller Erfolg gewertet werden. Was die konkrete thematische Zielsetzung betrifft so ist aus ITA-Sicht das Ziel einer expliziten Befassung mit der Situation "Entscheidung unter Ungewissheit" nicht ganz erreicht worden. Offenbar ist die Versuchung doch zu groß diesem Ungemach zu entweichen: Sei es durch den Verweis auf Forschungslücken und die anschlieBende Forderung nach mehr Forschung, sei es durch das Hervorheben von bestehendem Wissen und bereits erzielten Forschungsergebnissen, sei es auch nur durch den Hinweis, dass ohnehin schon viele Aktivitäten auf diesem Gebiet unternommen werden.

Das Programm sowie die Folien der Vorträge sind unter www.nanotrust.ac.at/nano10/ zu finden.

1 www.dguv.de/ifa/en/fac/nanopartikel/ beurteilungsmassstaebe/index.jsp.

2 www.bmu.de/files/pdfs/allgemein/application/pdf/ nanokomm_abschlussbericht_2008.pdf.

Ulrich Fiedeler 


\section{Lernen für die Transformation: Neue Perspektiven in der technische Ausbildung}

\author{
Nachhaltige Entwicklung erfordert, dass \\ die Gesellschaft die menschlichen Grund- \\ bedürfnisse und -rechte sowie die Gren- \\ zen der natürlichen Systeme besser be- \\ rücksichtigt. Vom 19.-22.9. befassten sich \\ etwa 150 Teilnehmerlnnen aus aller Welt \\ im Rahmen der internationalen Konfe- \\ renz EESD 2010 in Göteborg mit dem \\ Thema „Transformation technischer Aus- \\ bildung".
}

EESD 2010 war die fünfte Konferenz einer Serie von internationalen Tagungen, die alle zwei Jahre zum wissenschaftlichen Austausch über nachhaltige Entwicklung in technischer Ausbildung stattfinden. Heuer wurde die Konferenz von der Chalmers University in Göteborg und der KTH in Stockholm organisiert.

Ein Diskussionsthema für die Transformation sowohl aus der Perspektive der Lehre als auch in Hinblick auf Forschung und Praxis ist Inter-, und Transdisziplinarität. Einige interdisziplinäre Forschungsgruppen präsentierten ihre Erfahrung mit Lehrveranstaltungen für unterschiedliche Disziplinen, in denen Studierende dabei die gegensätzlichen Standpunkte bei der Entwicklung von technischen Lösungen kennenlernen können. Auch nicht-technische Fähigkeiten, wie etwa Kompromisse zu schließen, werden dabei gefördert.

Die Verknüpfung von Forschung und Lehre benötigt jedoch neve pädagogische Konzepte für die Vermittlung von Theorie und Praxis. Dieser Lernprozess nutzt dabei die unterschiedlichen Ausgangspunkte der Studierenden auf technischen, natur- und sozialwissenschaftlichen Gebieten. Die Gruppendynamik wird durch inhaltliche und organisatorische Interaktionen (auch Konflikte) zwischen Studierenden verschiedener Disziplinen und Länder beeinflusst. Die Erfahrung zeigt, dass vor allem die Erwartungen bezüglich der Ergebnisse von Lernprozessen sehr unterschiedlich sein können. In einigen Universitäten werden deshalb bei der Einführung der jeweiligen Lehrveranstaltung die Lernziele diskutiert und der Arbeitsaufwand gemeinsam mit den Studierenden und externen Fachleuten abgeschätzt.
Großer Wert wird auf die Rückmeldungen während Veranstaltungen, die Synthese der Ergebnisse und deren Evaluierung in verschiedenen Phasen der Arbeit gelegt. Beispiele aus Irland, Schweden und den Niederlanden konnten zeigen, dass die persönlichen Interessen der Studierenden die Qualität der Zusammenarbeit sowie die Ergebnisse der Arbeit entscheidend beeinflussen. Die Auseinandersetzung mit realen Problemstellungen kann dabei motivierend und inspirierend wirken.

Die EESD 2010 bot ein Diskussionsforum für unterschiedliche Erwartungen und Konzepte. In der Regel wird z. B. erwartet, dass in einer Lehrveranstaltung die kritische Auseinandersetzung mit dem Thema im Vordergrund steht, ohne ein unmittelbar umsetzbares Ergebnis zu erwarten.

Die Integration von Forschung und Lehre stellt hier aber neue Anforderungen an die Lehre. Durch die Projektarbeit in einer interdisziplinären Lehrveranstaltung muss oft ein umsetzbares Ergebnis im vereinbarten Zeitrahmen erreicht werden. Die Studierenden und Lehrenden werden dabei gemeinsam in die Verantwortung genommen. Die Entwicklung der Rahmenbedingungen für solche Mitverantwortung und die Weiterbildung der Lehrenden für neve Formen des Unterrichts waren Diskussionspunkte in der Konferenz. Außerdem wurde in Workshops über die Entwicklung never Unterrichtsmethoden und Textbücher für die "Transformation technischer Ausbildung" lebhaft diskutiert.

Das ITA war an der EESD 2010 durch die Mitwirkung im wissenschaftlichen Komitee, bei der Konzeption der Schlüsselfragen von Workshops, durch ein Keynote-Referat zum Thema "Technische Universitäten in lernenden Netzwerken" sowie mit einen Beitrag zum Thema "Umweltmanagement in der technischen Ausbildung" vertreten.

Weitere Informationen: EESD10.org.

Mahshid Sotoudeh 


\section{Aktuelle Publikationen}

\section{Referierte Beiträge}

Böschen, S., Kastenhofer, K., Rust, I., Soentgen, J. and Wehling, P., 2010, Scientific Nonknowledge and Its Political Dynamics: The Cases of Agri-Biotechnology and Mobile Phoning, Science, Technology, and Human Values 35(6), 783-811.

Čas, J., 2011 (forthcoming), Ubiquitous computing, privacy and data protection: options and limitations to reconcile the unprecedented contradictions, in: S. Gutwirth et al. (Hg.): Privacy and data protection : an element of choice, Springer.

\section{Artikel/Buchbeiträge}

Aichholzer, G., Bora, A., Bröchler, S., Decker, M., Latzer, M. (Hg.), 2010, Technology Governance. Der Beitrag der Technikfolgenabschätzung, Berlin: edition sigma.

Aichholzer, G., Strauß, S., 2010, Beteiligung von Bürger(inne)n an lokaler Klimaschutzpolitik. Untersuchung traditioneller und elektronischer Partizipationsformen, GAIA 19(4): 308-10 (im Erscheinen).

Aichholzer, G., Bora, A., Bröchler, S., Decker, M., Latzer, M., 2010, Einleitung und Überblick, in: Aichholzer, G., Bora, A., Bröchler, S., Decker, M., Latzer, M. (Hg.), Technology Governance. Der Beitrag der Technikfolgenabschätzung, Berlin: edition sigma, 13-28.

Fiedeler, U., Simkó, M., Gazsó, A., 2010, Governance von Nanotechnologie - Das österreichische Projekt NanoTrust als Beispiel, in: Aichholzer, G., Bora, A., Bröchler, S., Decker, M. and Latzer, M. (Eds): Technology Governance. Der Beitrag der Technikfolgenabschätzung, Berlin: edition sigma, 247-255.
Kastenhofer, K., 2010, Risk assessment of emerging technologies and post-normal science, Science, Technology, and Human Values, On-line first.

Peissl, W., 2010, Privacy in Europe from a TA perspective, in: Gutwirth, S., Poullet, Y. und Paul De Hert (Hg.): Data Protection in a Profiled World, Proceedings CPDP 2009, Berlin: Springer, 247-256.

Kubicek, H., Aichholzer, G., Allhutter, D., Cimander, R., Pina, V., Strauß, S., Torres, L., 2010, Comparative Evaluation of the Impact of E-participation in Local Climate Change Policy Programs, in: Chappelet, J.-L., Glassey, O., Janssen, M., Macintosh, A., Scholl, J., Tambouris, E., Wimmer, M. A. (eds.), Electronic Government and Electronic Participation. Joint Proceedings of Ongoing Research and Projects of IFIP EGOV and ePart 2010, Linz: Trauner, 377-382.

Ornetzeder M., Schrever, A., Rohracher H., 2010, Auf der Suche nach nachhaltigen Anwendungsformen der Brennstoffzellentechnologie: Erfahrungen mit Constructive Technology Assessment, in: Aichholzer, G, et al. (Hg.), Technology Governance. Der Beitrag der Technikfolgenabschätzung, edition sigma, Berlin, S. 271-278.

Nentwich, M., 2010, Web 2.0 and academia, in: Karner, S. und Getzinger, G. (Hg.): Proceedings of the 9th IAS-STS Annual Conference on Critical Issues in Science and Technology Studies, $2^{\text {nd }}-4^{\text {th }}$ May 2010, Graz, Austria, Graz: CDROM, 66-78. 


\section{Bücher/Herausgeberschaften}

Aichholzer, G., Bora, A., Bröchler, S., Decker, M., Latzer, M. (Hg.), 2010, Technology Governance. Der Beitrag der Technikfolgenabschätzung, Berlin: edition sigma.

\section{Forschungsberichte}

Eisenberger, I., Nentwich, M., Fiedeler, U., Gazsó, A., Simkó, M., 2010, Nano Regulation in the European Union, NanoTrustDossiers, Nr. 017en - November 2010, Wien: Institut für Technikfolgen-Abschätzung (ITA) [http://epub.oeaw.ac.at/ita/ nanotrust-dossiers/dossier017en.pdf].

EPTA, 2010, Routes to sustainable transport. An overview of status and policy strategies for sustainable transport in Europe. Report by the European Parliamenatary Technology Assessment (EPTA) network, Copenhagen, 26 October

[http://epub.oeaw.ac.at/ita/ita-projektberichte/ EPTA-TransportReport_Oct2010.pdf]

[http://www.tekno.dk/EPTA/documents/2010/ p10_EPTA_routes_to_sustainable transport.pdf].

Fries, R., Greßler, S., Simkó, M., Gazsó, A., Fiedeler, U., Nentwich, M., 2010, Nanosilver, NanoTrust-Dossiers, Nr. 010en - November 2010, Wien: Institut für Technikfolgen-Abschätzung (ITA) [http://epub.oeaw.ac.at/ita/ nanotrust-dossiers/dossier010en.pdf].
Lindner, R., Beckert, B., Goos, K., Hennen, L., Aichholzer, G., Strauß, S., 2010, E-Democracy in Europe - Prospects of Internet-based political participation. Theoretical framework and overview: in-depth examination of three selected areas - Phase II, Deliverable No. 2, Science and Technology Options Assessment (STOA), European Parliament, Brussels.

Simkó, M., Nentwich, M., Gazsó, A., Fiedeler, U., 2010, How Nanoparticles Enter the Human Body and Their Effects There, NanoTrust-Dossiers, Nr. 003en - November 2010, Wien: Institut für Technikfolgen-Abschätzung (ITA) [http://epub.oeaw.ac.at/ita/ nanotrust-dossiers/dossier003en.pdf].

Torgersen, H., Schmidt, M., Kastenhofer, K., 2010, Engineering of living artefacts: synthetic biology. pp 11-50 in: Science and Technology Options Assessment STOA, Making Perfect Life - Bio-Engineering (in) the $21^{\text {st }}$ Century. Interim Study, Monitoring Report, IP/A/STOA/FWC-2008-96/LOT6/ $\mathrm{SCl}$, Brussels: European Parliament.

Torgersen H., Cserer A., Doblhammer S., Giersch G., Gschmeidler B., Holtz P., Kerbe W., Kronberger N., Meinhart C. Schmidt M., Seiringer A., Strasser E., Wagner W., Waigmann E., 2010, Communicating Synthetic Biology (COSY), a GEN-AU/ELSA project, Final Report to the FFG, Vienna: ITA.

\section{Konferenzbeiträge/Vorträge}

Aichholzer, G., Allhutter, D., Strauß, S., 2010, Differences between Effects of Online and Offline Participation? Presentation at the 2nd International Conference on eParticipation (ePart 2010), University of Lausanne/ Switzerland, 31 st August.
Aichholzer, G., 2010, Anmerkungen zur Onlinevariante der Europäischen Bürgerinitiative, Roundtable Europäische Bürgerinitiative: Rechtsetzung durch europäische Zivilgesellschaft? EU-Informationsveranstaltung "Rechtsinformatik", Parlament, Wien, 23. September. 
Aichholzer, G., Strauß, S., 2010, Staatliches Identitätsmanagement als Systeminnovation. Ein tragfähiger Ansatz zur Gestaltungsund Folgenanalyse? NTA4 2010 - Vierte Konferenz des Netzwerks TA: „Der Systemblick auf Innovation - Technikfolgenabschätzung in der Technikgestaltung", Berlin, 24.26. November.

Allhutter, D. 2010, Rasse' und ,Ethnizität' als Fetisch in computergenerierter Pornografie, C3-Centrum für Internationale Entwicklung, Wien, 23. September 2010.

Allhutter, D. 2010, Geschlechterdifferenz, Heterornomativität und rassisierende Strategien in der Herstellung digitaler Pornografie, Keynote im Rahmen der Konferenz „Pornografisierung von Gesellschaft?!", 28-30 Oktober 2010, Fachhochschule Köln.

Allhutter, D., 2010. Wie Geschlechter gemacht werden: die Programmierung von computergenerierten Genitalien und sexuellem Handlungsrepertoire, eingeladener Vortrag im Rahmen des Kolloquiums "Wissenschaft, Technik und Körper III", TU Braunschweig, 2. November 2010.

Allhutter, D., 2010, Digitale Pornografie als technowissenschaftliches Forschungsobjekt, eingeladener Vortrag im Rahmen der Ringvorlesung 'Sind wir nie modern gewesen? Gender in der technologisierten Leistungsgesellschaft', Universität Wien, 9. November 2010 .

Allhutter, D., 2010, Computerunterstütze PornoBastelei-Differenz als Techno-Warenfetisch, eingeladener Vortrag am IWK-Institut für Wissenschaft und Kunst, Wien, 10. November 2010.

Čas, J., 2010, Informationsgesellschaft und IKT, Keynote-Vortrag bei der Tagung "Informationsgesellschaft und Infrastrukturpolitik im Alpenraum", Hochschule für Technik und Wirtschaft HTW, 23. September, Chur.

Fiedeler, U. and Torgersen, H., 2010, If it is risky, it must be real-How risk discourses co-constitute nanotechnology, ScieCom 2010, 11. 11.2010, Wien.
Gudowsky, N., Bechtold, U. und Wilfing, H., 2010, Information and public opinion - comparing outcomes of an organized participatory approach and a street questioning on economic issues of global warming, Posterpresentation, Advancing Sustainability in a Time of Crisis (ISEE Conference 2010), 22.25. August, Oldenburg \& Bremen.

Kastenhofer, K., 2010, What happens to interdisciplinarity when disciplines become extinct? On interdisciplinarity in a technoscientific context, workshop "Philosophy of/as Interdisciplinarity", 18.-21. Sept., Udo-KellerStiffung, Hamburg.

Kastenhofer, K., 2010, Die neuen Technowissenschaften: hybride Praktiken, GovernanceRegimes oder performative Diskurse?, Vortrag im Rahmen der Ringvorlesung 'Sind wir nie modern gewesen? Gender in der technologisierten Leistungsgesellschaff', Universität Wien, 9. November 2010.

Kastenhofer, K., 2010, Was passiert mit Wissenschaftskulturen, wenn Disziplinengrenzen obsolet werden? Interdisziplinarität im techno-wissenschaftlichen Kontext, Vortrag am Forschungstag "Wissenschaftskulturen", 17. Nov. 2010, Univ. Klagenfurt.

Ornetzeder, M., 2010, Sozio-technische Szenarien und Kernhandlungsfelder für ein nachhaltiges Energiesystem in Österreich, Vierte Konferenz des "Netzwerks TA" (NTA4), Der Systemblick auf Innovation - Technikfolgenabschätzung in der Technikgestaltung, Berlin, 25. November.

Peissl, W., 2010, Digitale Welt - Privatsphäre und Technikgestaltung: der Beitrag der Technikfolgenabschätzung, Gastvortrag auf der Generalversammlung der IEEE Austria, 14. Oktober., Wien.

Peissl, W., 2010, Sicherheit und Datenschutz als integrierte Planungsvorgaben von Produktund Technologieentwicklung, Vortrag auf der Jahreskonferenz des Instituts für Sicherheitsforschung an der Sigmund Freud Privat Universität Wien im Rahmen des KIRASProjekts SFI@SFU, 22. Oktober, Wien. 
Sotoudeh, M., 2010, Understanding of technology development as a social process in learning networks. Engineering Education in Sustainable Development, Keynote 20.09., Göteborg, Chalmers University \& KTH.

Sotoudeh, M., Gazsó A.,2010, Analysis of a project oriented training seminar for Eco Management Audit Scheme (EMAS) in Austria Learning by doing for engineers, Engineering Education in Sustainable Development, 21.09., Göteborg, Chalmers University \& $\mathrm{KTH}$.

Schmidt, J. C. and Kastenhofer, K., 2010, Beyond nature and culture: (Baconian) technosciences and "their power to change the world", Second Annual Conference of the Society for the Study of Nanoscience and Emerging Technologies, 29. Sept.-2. Okt., Technische Universität Darmstadt.

\section{Sonstiges/Kurzbeiträge}

Allhutter, D. 2010, Infrastrukturen digitaler Pornografie. an.schläge. Juni 2010: p. 20.

Allhutter, D., 2010, Ver/Behinderung, Kurzrezension 'Gendering Disability. Intersektionale Aspekte von Behinderung und Geschlecht. Hg. von Jutta Jacob, Swantie Köbsell und Eske Wollrad. transcript, Bielefeld 2010'. Weiberdiwan. Die feministische Rezensionszeitschrift, Herbst/Winter 2010, 5.
Simkó M.: Cells meet electromagnetic fields: Cell type and redox status dependent effects. EMF symposium, "Low frequency electromagnetic field exposure and modulation of cellular functions". 16.09.2010, Wageningen (NL) .

Wächter, P., Ornetzeder, M., Schrever, A., 2010 , Critical issues in the spatial organisation in the transition to a sustainable energy system. Advances in Energy Studies 2010, 19 th $^{\text {- } 21^{\text {st }} \text { October, Barcelona. }}$

Wächter, P. 2010, Critical issues in the spatial organisation in the transition to a sustainable energy system. Poster presentation, Advances in Energy Studies 2010, 19th $-21^{\text {st }}$ October 2010, Barcelona.

Wächter, P., 2010, Räumliche Aspekte eines nachhaltigen Energiesystems - Backcasting als Methode für Systeminnovation. Vortrag, 4. Konferenz des Netzwerks Technikfolgen-Abschätzung, 24.-26.11.2010, Berlin.

Ornetzeder, M., 2010, Praxisnaher Wissenserwerb. Eine Lehrveranstaltung als "Wissenschaftliche Konferenz", BOKU INSIGHT, Zeitschrift der Universität für Bodenkultur, Nr. 4, September, Wien, S. 14-15

[http://www.boku.ac.at/insightlehre.html].

Strauß, S., 2010, Innovationsnetzwerke und "Real-World"-Experimente [NL-Schwerpunkt: Forschung und Technologieanwendung als gesellschaftliche Praktiken],

[http://epub.oeaw.ac.at/ita/ ita-newsletter/NL0910.pdf\#4]. 


\section{Aktuelle Buchpublikationen des ITA}

Technology Governance. Der Beitrag der Technikfolgenabschätzung.

Aichholzer, G., Bora, A., Bröchler, S., Decker, M., Latzer, M. (Hg.), 2010, Berlin: edition sigma

From Need to Greed. The Changing Role of Technology in Society.

Ernest Braun, 2010, Austrian Acadmy of Sciences Press, Vienna. 154 pp.

[http://epub.oeaw.ac.at/6916-1]

Understanding Nanotechnology: Philosophy, Policy and Publics.

Ulirch Fiedeler, Coenen, C., Davies, S. R. and Ferrari, A. (eds.), 2010, Heidelberg: Akademische Verlagsgesellschaft AKA.

Inter- und Transdisziplinarität im Wandel? Neue Perspektiven auf problemorientierte Forschung und Politikberatung.

Bogner, A., Kastenhofer, K. und Torgersen, H. (Hg.)

in Reihe: Wissenschafts- und Technikforschung,

hg. v. Bora, A., Maasen, S., Reinhardt, C. und Wehling, P.,

2010, Baden-Baden: nomos.

Handbuch Strategische Umweltprüfung - Die Umweltprüfung von Politiken, Plänen und Programmen. 3., erweiterte Auflage Dezember 2009

ITA/Ulrike Bechtold (Hg.), Kerstin Arbter (Autorin)

Verlag der Österreichischen Akademie der Wissenschaften: Wien

[http://epub.oeaw.ac.at/Handbuch-SUP].

Interviewing Experts.

Bogner, A., Littig, B., Menz, W. (Hrsg.)

2009, Palgrave Macmillan, Basingstoke

Experteninterviews. Theorien, Methoden, Anwendungsfelder.

Bogner, A., Littig, B., Menz, W. (Hrsg.), 3. grundlegend überarb. Aufl. 2009, Verlag für Sozialwissenschaften, Wiesbaden

Technical education for sustainability. An analysis of needs in the $21^{\text {st }}$ century.

Sotoudeh, M., in Reihe: Environmental Education, Communication and

Sustainability, Bd. 30, hg. v. Filho, W. L.,

2009, Peter Lang Internationaler Verlag der Wissenschaften,

Frankfurt am Main

Nähere Informationen unter: www.oeaw.ac.at/ita/books.htm

Impressum:

Medieninhaber:

Österreichische Akademie der Wissenschaften

Juristische Person öffentlichen Rechts (BGBI 569/1921

idF BGBI I 130/2003)

Dr. Ignaz Seipel-Platz 2, A-1010 Wien

Herausgeber:

(C) Institut für Technikfolgen-Abschätzung (ITA)

Strohgasse 45/5, A-1030 Wien

Alle Rechte vorbehalten.

Der ITA-Newsletter erscheint viermal jährlich und enthält Informationen über nationale und internationale Trends in der Technikfolgen-Abschätzung, ITA-Forschungsprojekte, Publikationen der ITA-MitarbeiterInnen und ITA-Veranstaltungen. Das ITA verfolgt mit diesem periodischen Medium das Ziel, wissenschaftliche Zusammenhänge zwischen Technik und Gesellschaft einem breiten Leserlnnenkreis zugänglich zu machen. 
ITA-Seminar: 11. Jänner 2011, 16:00-18:00 Uhr

DI Dr. Harald Frey

TU Wien, Forschungsbereich für Verkehrsplanung und Verkehrstechnik

\section{Elektromobilität als Lösung der Verkehrsprobleme? - Chancen und Grenzen "}

Ort: Bibliothek des ITA, 1030 Wien, Strohgasse 45, 3. Stock, Tür 5

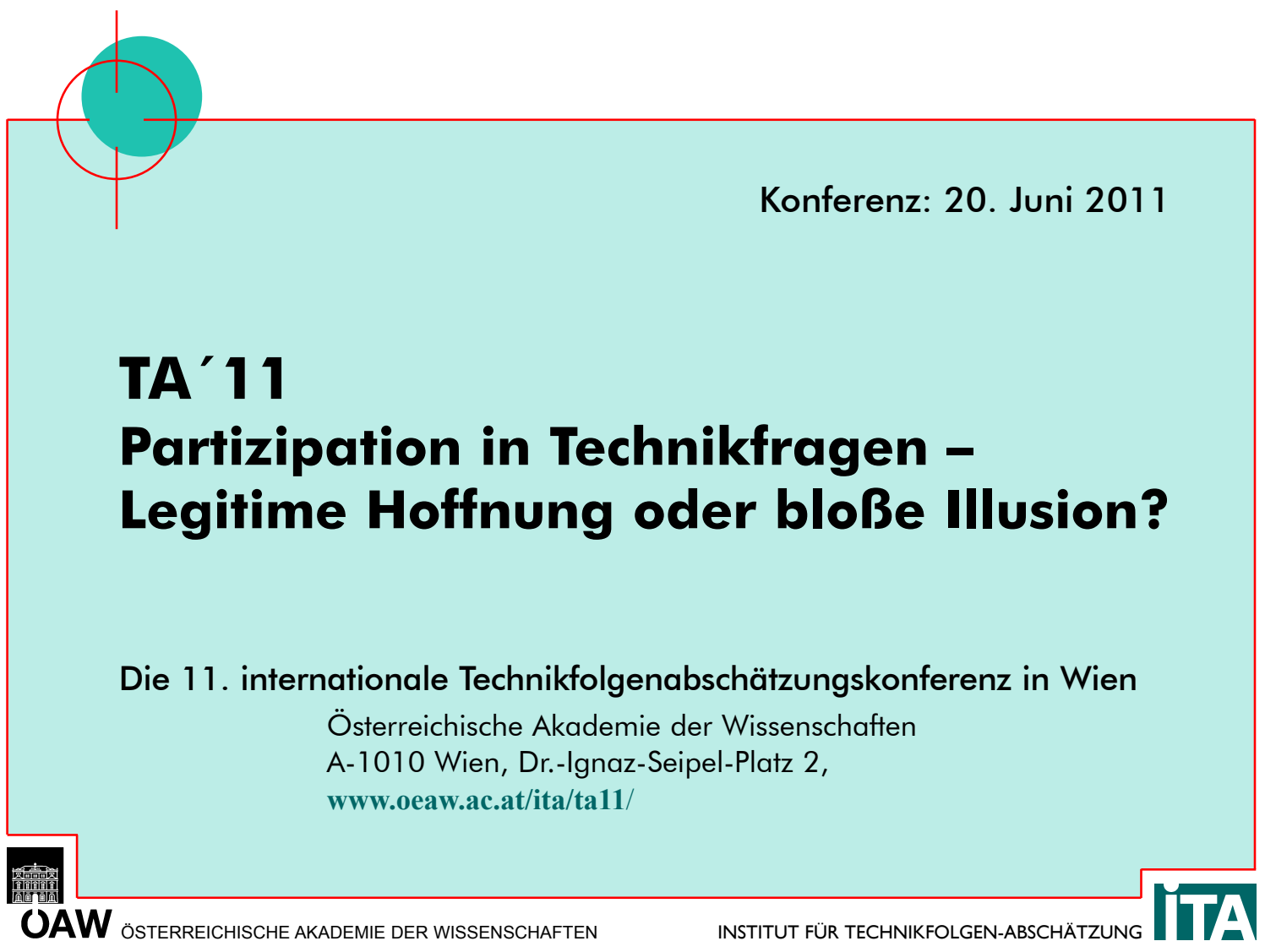


Die 1 News werden herausgegeben vom Institut für Technikfolgen-Abschätzung der Österreichischen Akademie der Wissenschaften (ITA). Für weiterführende Fragen zu den in dieser Ausgabe behandelten Themen und zur Technikfolgen-Abschätzung im Allgemeinen stehen wir Ihnen gerne zur Verfügung:

Institut für Technikfolgen-Abschätzung (ITA)

der Österreichischen Akademie der Wissenschaften

A-1030 Wien, Strohgasse 45/5/3. Stock

Tel.: +43-1-51581/6582, Fax: +43-1-710 9883

E-Mail: tamail@oeaw.ac.at, www.oeaw.ac.at/ita

Leiter des Instituts:

Univ.-Doz. Mag. Dr. Michael NENTWICH ..DW 6583.....mnent@oeaw.ac.at MitarbeiterInnen:

Mag. Dr. Georg AICHHOLZER .DW6591.....aich@oeaw.ac.at Mag.a Dr.in Doris ALLHUTTER DW 6585.....dallhutt@oeaw.ac.at

Mag.a Dr.in Ulrike BECHTOLD .DW 6593.....ubecht@oeaw.ac.at

Dipl.-Soz. Dr. Alexander BOGNER DW 6595.....abogner@oeaw.ac.at

Ing. Mag. Johann ČAS .DW6581.....jcas@oeaw.ac.at

Mag. Alex DEGELSEGGER .DW 6595.....adegel@oeaw.ac.at

Dipl.-Phys. Dr. Ulrich FIEDELER .DW 6577.....ufiedeler@oeaw.ac.at

MMag. Dr. André GAZSÓ .DW 6578.....agazso@oeaw.ac.at

Mag. Niklas GUDOWSKY .DW 6593.....niklas.gudowsky@oeaw.ac.at

Mag.a Julia HASLINGER .DW6594....jhasl@oeaw.ac.at

Mag.(FH) Werner KABELKA .DW 6587.....kabelka@oeaw.ac.at

Mag.a Dr.in Karen KASTENHOFER .DW6580.....kkast@oeaw.ac.at

Mag. René KÖNIG DW 6597.....rene.koenig@assoc.oeaw.ac.at

Mag. Dr. Michael ORNETZEDER DW6589.....ornetz@oeaw.ac.at

Mag. Dr. Walter PEISSL DW 6584.....wpeissl@oeaw.ac.at

Barbara POPPEN .DW 6582.....bpoppen@oeaw.ac.at

Prof.in Dr.in Myrtill SIMKÓ .DW 6579.....msimko@oeaw.ac.at

PDin Dlin Dr.in Mahshid SOTOUDEH DW 6590.....msotoud@oeaw.ac.at

Sabine STEMBERGER .DW6586.....sstem@oeaw.ac.at

Mag. Stefan STRAUSS .DW 6599.....sstrauss@oeaw.ac.at

Dr. Helge TORGERSEN .DW6588.....torg@oeaw.ac.at Mag.a Petra WÄCHTER DW6592.....pwaecht@oeaw.ac.at

E-Mail-Newsservice: Wenn Sie an Berichten, Newslettern, Veranstaltungshinweisen etc. interessiert sind, registrieren Sie sich bitte unter https://lists.oeaw.ac.at/mailman/listinfo/itanews.

ITA auf Twitter: twitter.com/technikfolgen

ITA auf Facebook: facebook.com/Institute.of.technology.assessment 